\title{
Ethnic differences in the incidence of clinically diagnosed
}

\section{influenza: an England population-based cohort study 2008-}

\section{8 [version 1; peer review: 1 approved]}

\author{
Jennifer Davidson (iD)1, Amitava Banerjee2, Rohini Mathur (iD)1, Mary Ramsay (iD)3,4, \\ Liam Smeeth (iD)1,4, Jemma Walker ${ }^{4-6}$, Helen McDonald (D) 4,5*, \\ Charlotte Warren-Gash (D) 1*
}

\footnotetext{
${ }^{1}$ Department of Non-communicable Disease Epidemiology, London School of Hygiene and Tropical Medicine, London, UK

2Institute of Health Informatics, University College London, London, UK

${ }^{3}$ Immunisation and Countermeasures, National Infection Service, Public Health England, London, UK

${ }^{4}$ National Institute for Health Research Health Protection Research Unit in Immunisation, London School of Hygiene and Tropical Medicine in partnership with Public Health England, London, UK

${ }^{5}$ Department of Infectious Disease Epidemiology, London School of Hygiene and Tropical Medicine, London, UK

${ }^{6}$ Statistics, Modelling and Economics Department, Public Health England, London, UK

* Equal contributors
}

V1 First published: 04 Mar 2021, 6:49

https://doi.org/10.12688/wellcomeopenres.16620.1

Latest published: 04 Mar 2021, 6:49

https://doi.org/10.12688/wellcomeopenres.16620.1

\section{Abstract}

Background: People of non-White ethnicity have a higher risk of severe outcomes following influenza infection. It is unclear whether this is driven by an increased risk of infection or complications. We therefore aimed to investigate the incidence of clinically diagnosed influenza/influenza-like illness (ILI) by ethnicity in England from 20082018.

Methods: We used linked primary and secondary healthcare data (from the Clinical Practice Research Datalink [CPRD] GOLD and Aurum databases and Hospital Episodes Statistics Admitted Patient Care [HES $A P C])$. We included patients with recorded ethnicity who were aged 40-64 years and did not have a chronic health condition that would render them eligible for influenza vaccination. ILI infection was identified from diagnostic codes in CPRD and HES APC. We calculated crude annual infection incidence rates by ethnic group. Multivariable Poisson regression models with random effects were used to estimate any ethnic disparities in infection risk. Our main analysis adjusted for age, sex, and influenza year.

Results: A total of 3,735,308 adults aged 40-64 years were included in the study; $87.6 \%$ White, $5.2 \%$ South Asian, 4.2\% Black, 1.9\% Other, and $1.1 \%$ Mixed. We identified 102,316 ILI episodes recorded among 94,623 patients. The rate of ILI was highest in the South Asian (9.6 per 1,000 person-years), Black (8.4 per 1,000 person-years) and Mixed (6.9

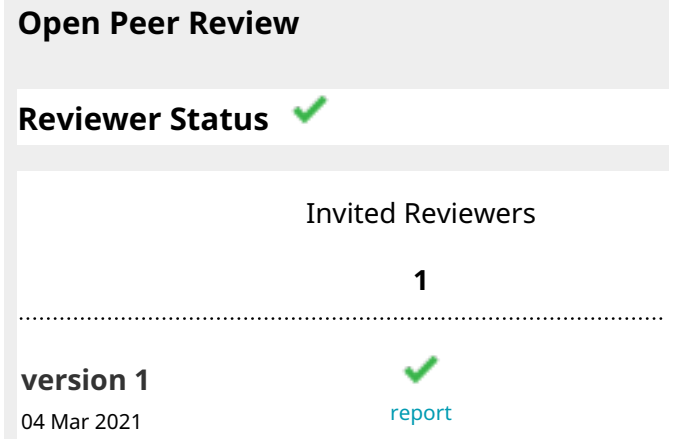

1. Benjamin J. Cowling (iD, The University of Hong Kong, Hong Kong, China

Any reports and responses or comments on the article can be found at the end of the article. 
per 1,000 person-years) ethnic groups. The ILI rate in the White ethnic group was 5.7 per 1,000 person-years. After adjustment for age sex and influenza year, higher incidence rate ratios (IRR) for ILI were seen for South Asian (1.70, 95\% CI 1.66-1.75), Black (1.48, 1.44-1.53) and Mixed $(1.22,1.15-1.30)$ groups compared to White ethnicity. Conclusions: Our results suggest that influenza infection risk differs between White and non-White groups who are not eligible for routine influenza vaccination.

\section{Keywords}

ethnicity, inequalities, influenza, respiratory infection

Corresponding author: Jennifer Davidson (Jennifer.Davidson@lshtm.ac.uk)

Author roles: Davidson J: Data Curation, Formal Analysis, Methodology, Writing - Original Draft Preparation, Writing - Review \& Editing; Banerjee A: Writing - Review \& Editing; Mathur R: Methodology, Writing - Review \& Editing; Ramsay M: Conceptualization, Writing Review \& Editing; Smeeth L: Writing - Review \& Editing; Walker J: Methodology, Writing - Review \& Editing; McDonald H: Conceptualization, Methodology, Writing - Original Draft Preparation, Writing - Review \& Editing; Warren-Gash C: Methodology, Supervision, Writing - Original Draft Preparation, Writing - Review \& Editing

Competing interests: All authors have completed the ICMJE uniform disclosure form (www.icmje.org/coi_disclosure.pdf). A.B. has received grants from Astra Zeneca, UK Research and Innovation (UKRI), and the NIHR. R.M. has received grants from the Wellcome Trust and personal fees from Amgen. M.R. reports that Public Health England Immunisation and Countermeasures Division has provided vaccine manufacturers with post-marketing surveillance reports on vaccine-preventable infection which the companies are required to submit to the UK Licensing Authority in compliance with their Risk Management Strategy, and a cost recovery charge is made for these reports. L.S. has received grants from the NIHR, the Wellcome Trust, Glaxo-Smith Kline, the British Heart Foundation, Diabetes UK, the Newton Fund, and UKRI; he is also a non-executive director of the Medicines and Healthcare products Regulatory Agency (MHRA). C.W.G. has received speaker fees from Sanofi Pasteur.

Grant information: This work was supported in part by the British Heart Foundation [FS/18/71/33938] who fund a Non-Clinical PhD Studentship for J.D., the Wellcome Trust [201440/Z/16/Z] who fund an Intermediate Clinical Fellowship for C.W.-G, and the National Institute for Health Research (NIHR) Health Protection Research Unit (HPRU) in Immunisation at the London School of Hygiene and Tropical Medicine in partnership with Public Health England, who fund J.W., H.M., L.S. and M.R. The funders had no role in study design, data collection and analysis, preparation of the manuscript, or the decision to publish. The views expressed are those of the authors and not necessarily those of the NHS, the NIHR, the Department of Health and Social Care, or Public Health England.

The funders had no role in study design, data collection and analysis, decision to publish, or preparation of the manuscript.

Copyright: $\odot 2021$ Davidson J et al. This is an open access article distributed under the terms of the Creative Commons Attribution License, which permits unrestricted use, distribution, and reproduction in any medium, provided the original work is properly cited.

How to cite this article: Davidson J, Banerjee A, Mathur R et al. Ethnic differences in the incidence of clinically diagnosed influenza: an England population-based cohort study 2008-2018 [version 1; peer review: 1 approved] Wellcome Open Research 2021, 6:49 https://doi.org/10.12688/wellcomeopenres.16620.1

First published: 04 Mar 2021, 6:49 https://doi.org/10.12688/wellcomeopenres.16620.1 


\section{Introduction}

People from ethnic minority backgrounds are represented disproportionately among patients with severe coronavirus disease 2019 (COVID-19). Recent research has found people of Black and South Asian ethnicity have increased risk of severe acute respiratory syndrome coronavirus 2 (SARS-CoV-2) infection, COVID-19-related hospitalization and death, independent of deprivation, occupation, household size, and underlying health conditions ${ }^{1,2}$.

The COVID-19 pandemic has reinforced the importance of seasonal influenza vaccination. By preventing influenza-related hospitalization, vaccination can minimize the risk of hospitalacquired COVID-19 (co-) infection for these individuals and reduce health service pressures, particularly the need for isolation of patients with respiratory symptoms awaiting COVID-19 test results.

In the United Kingdom (UK), the influenza vaccine is routinely recommended for adults aged $\geq 65$ years, or people $<65$ years with underlying health conditions. These recommendations formed the basis of the original guidance to identify patients at moderate- and high-risk of COVID-19. Influenza vaccine recommendations were expanded for the 2020/21 season to include all adults $\geq 50$ years $^{3}$. However, vaccine uptake among clinical risk groups is low, particularly for Black and Mixed Black ethnic groups ${ }^{4}$. In addition, people of non-White ethnicity have higher risk of severe outcomes following influenza infection $^{5,6}$. It is unclear whether this is driven by the risk of infection or complications, with most research focused on distal outcomes rather than initial infection risk.

Here we investigate the incidence of influenza and influenza-like illness (ILI) by ethnicity from 2008-2018 among people not eligible for routine influenza vaccination, to consider disparities in infection risk.

\section{Methods}

\section{Study design and data sources}

We conducted a retrospective cohort study using anonymized primary care data from the UK Clinical Practice Research Datalink (CPRD) GOLD and Aurum databases ${ }^{7,8}$ with linked secondary care data from the Hospital Episodes Statistics Admitted Patient Care (HES APC) database and death data from the Office for National Statistics. The data include diagnoses, prescriptions, immunizations and demographics. The corresponding author had full access to all CPRD GOLD and Aurum data used in the study, with relevant linked patient HES APC and ONS death data obtained from CPRD.

\section{Study population}

We included all adults aged 40-64 years registered at a CPRD contributing practice in England between 01/09/2008 and 31/08/2018 who were present in the GOLD and Aurum datasets. We then excluded among this study population any patients with a health condition indicative of influenza vaccination eligibility (Table 1 ), and those who had ever received pneumococcal vaccination, or influenza vaccination in the
12 months before baseline (all codes listed here, DOI: awaiting). Among the final study population, we started study follow-up to identify diagnoses of ARI (outcome of interest) at the latest of 12 months after current registration, up-toresearch-standard (GOLD only), 40 $0^{\text {th }}$ birthday, or 01/09/2008. Follow-up ended at the earliest of; a new diagnosis of a condition conferring eligibility for vaccination, pneumococcal or influenza vaccination, death, transfer out, the practice's last data collection, 65th birthday, or 31/08/2018.

\section{Variables}

Self-reported ethnicity was captured in CPRD and supplemented with HES APC if missing in CPRD. We grouped ethnicity into the five and 16 census categories of White (British, Irish, Other White), South Asian (Indian, Pakistani, Bangladeshi, other Asian), Black (African, Caribbean, other Black), Other (Chinese, all other), and Mixed (White and Asian, White and African, White and Caribbean, Other Mixed).

Influenza/ILI infection was identified from diagnostic codes in CPRD and HES APC. In a second analysis, we expanded our outcome definition to acute respiratory infection (ARI), additionally including codes for pneumonia, acute bronchitis, or other acute infections suggestive of lower respiratory tract involvement (all codes listed here, DOI: https://doi.org/10.17037/ DATA.00002102)

\section{Statistical analysis}

All analyses were done with Stata (version 16). We calculated crude annual infection incidence rates by ethnic group with age- and sex-stratification. Multivariable Poisson regression models with random effects, to account for multiple infections in the same patient, were used to estimate any ethnic disparities in infection risk. Our main analysis adjusted for age, sex, and influenza season/year. A second model additionally adjusted for region of residence and socioeconomic status (based on patient-level Townsend score quintiles), which may both confound and mediate an association between ethnicity and infection.

An earlier version of this article can be found on medRxiv (https://doi.org/10.1101/2021.01.15.21249388).

\section{Results}

Our cohort included 3,735,308 patients (Figure 1), of whom $87.6 \%$ were White $(n=3,271,115), \quad 5.2 \%$ South Asian $(n=196,262), 4.2 \%$ Black $(n=157,075), 1.9 \%$ Other $(n=69,440)$, and $1.1 \%$ Mixed $(n=41,416)$ (Table 2$)$. We excluded $511,682(12.0 \%)$ patients with no recorded ethnicity; this group had longer follow-up, fewer consultations and were more likely to be male than the included study population (Table 2). 16-category ethnicity was known for 3,035,689 of the cohort (with HES ethnicity breakdown beyond white and mixed not available), of whom $76.3 \%$ were White British, $0.9 \%$ Irish, $7.7 \%$ Other White, 2.6\% Indian, $1.3 \%$ Pakistani, $0.4 \%$ Bangladeshi, 2.2\% Other Asian, 2.8\% African, 1.5\% Caribbean, $0.9 \%$ Other Black, $0.7 \%$ Chinese, and $1.5 \%$ Other (Table 3). Non-White populations were younger and resided in more deprived areas than the White population, while a higher proportion of the White population were obese. 
Table 1. Definitions used for developing exclusion conditions using Clinical Practice Research Datalink (CPRD) code lists.

\begin{tabular}{|c|c|}
\hline Health condition & Study definition \\
\hline Cardiovascular disease (CVD) & $\begin{array}{l}\text { Any previous clinical diagnosis, major intervention for, or clinical review specific to CVD including heart } \\
\text { disease (congenital or otherwise), heart failure, stroke or transient ischaemic attack. }\end{array}$ \\
\hline Chronic liver disease & $\begin{array}{l}\text { Any previous clinical diagnosis of, or clinical review specific to, chronic liver disease including cirrhosis, } \\
\text { oesophageal varices, biliary atresia and chronic hepatitis. }\end{array}$ \\
\hline Chronic kidney disease (CKD) & $\begin{array}{l}\text { Any previous clinical diagnosis of, or clinical review specific to, CKD stages 3-5, history of dialysis or renal } \\
\text { transplant in Gold or Aurum. Or with estimated glomerular filtration rate (eGFR) to classify CKD stage } \\
\text { 3-5 in Gold. }\end{array}$ \\
\hline Chronic respiratory disease & $\begin{array}{l}\text { Any previous clinical diagnosis of, or clinical review specific to, chronic respiratory disease, including } \\
\text { chronic obstructive pulmonary disease, emphysema, bronchitis, cystic fibrosis, or fibrosing interstitial } \\
\text { lung diseases. }\end{array}$ \\
\hline Asthma & $\begin{array}{l}\text { Any previous clinical diagnosis of, or clinical review specific to, asthma with at least two prescriptions of } \\
\text { inhaled steroids in the year before baseline. Or any previous hospitalisation for asthma. }\end{array}$ \\
\hline Chronic neurological disease & $\begin{array}{l}\text { Any previous clinical diagnosis of, or clinical review specific to, a neurological disease such as Parkinson's } \\
\text { disease, motor neurone disease, multiple sclerosis (MS), cerebral palsy, dementia or a learning/ } \\
\text { intellectual disability. }\end{array}$ \\
\hline Diabetes mellitus & $\begin{array}{l}\text { Any previous diagnosis of, or clinical review specific to, diabetes mellitus, or with a prescription for } \\
\text { medication used to treat diabetes. }\end{array}$ \\
\hline Asplenia/sickle cell disease & $\begin{array}{l}\text { Any previous clinical diagnosis of, or clinical review specific to, asplenia or dysfunction of the spleen } \\
\text { (including sickle cell disease but not sickle cell trait). }\end{array}$ \\
\hline Severe obesity & Latest body mass index before baseline was $\geq 40 \mathrm{~kg} / \mathrm{m}^{2}$. \\
\hline \multirow{4}{*}{ Immunosuppression } & $\begin{array}{l}\text { Any previous clinical diagnosis of, or clinical review specific to, HIV, solid organ transplant or other } \\
\text { permanent immunosuppression (such as genetic conditions compromising immune function). }\end{array}$ \\
\hline & $\begin{array}{l}\text { In the two years before baseline: clinical diagnosis of, or clinical review specific to, aplastic anaemia or } \\
\text { haematological malignancy, or receiving a bone marrow or stem cell transplant. }\end{array}$ \\
\hline & $\begin{array}{l}\text { In the year before baseline: previous clinical diagnosis of, or clinical review specific to, other/unspecified } \\
\text { immune deficiency or receiving chemotherapy or radiotherapy. }\end{array}$ \\
\hline & $\begin{array}{l}\text { In the year before baseline: prescription of biological therapy or at least } 2 \text { prescriptions for oral steroids } \\
\text { or other immunosuppressants including DMARDS, Methotrexate, Azathioprine, or corticosteroid } \\
\text { injections. }\end{array}$ \\
\hline
\end{tabular}

Met patient inclusion criteria
-With HES linked data
-Aged 40-64 years 01/09/2008-31/08/2018
-In CPRD follow-up 01/09/2008-31/08/2018
n=6,075,321

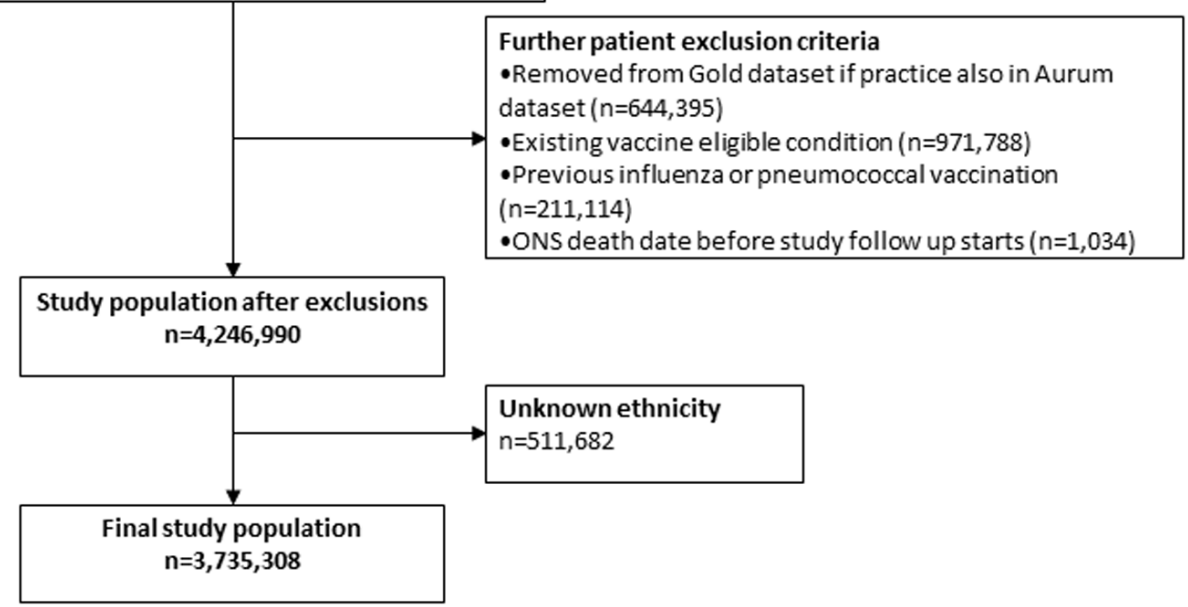

Figure 1. Study population flow chart. 


\begin{tabular}{|c|c|c|c|c|c|c|c|c|c|c|c|c|c|c|c|c|c|c|c|c|c|}
\hline 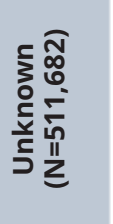 & 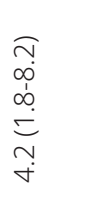 & 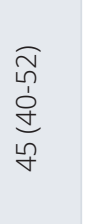 & & 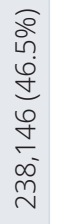 & 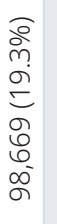 & 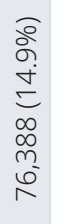 & 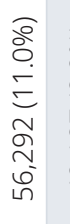 & 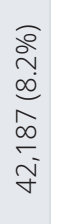 & & 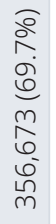 & 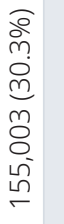 & & 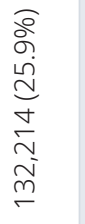 & 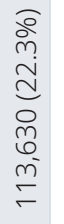 & 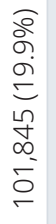 & 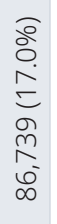 & 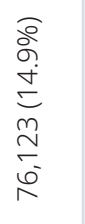 & & 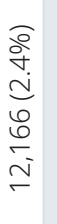 & 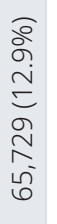 & 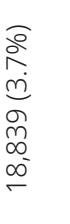 \\
\hline 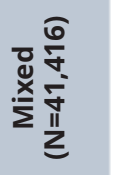 & 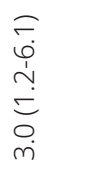 & $\begin{array}{l}\frac{g}{g} \\
\dot{g} \\
\stackrel{g}{y}\end{array}$ & & 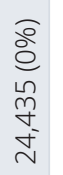 & 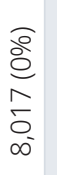 & 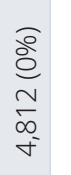 & 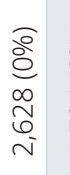 & 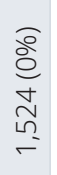 & & $\begin{array}{l}\overline{0} \\
0 \\
\overline{0} \\
\infty \\
0 \\
i\end{array}$ & 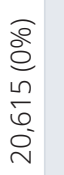 & & $\begin{array}{l}\bar{a} \\
\text { o } \\
\hat{8} \\
\text { i }\end{array}$ & 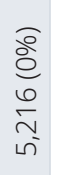 & $\begin{array}{l}\bar{a} \\
\text { o } \\
0 \\
0 \\
0 \\
0\end{array}$ & $\begin{array}{l}\bar{o} \\
\stackrel{0}{0} \\
\text { ô } \\
o \\
\infty\end{array}$ & 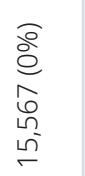 & & 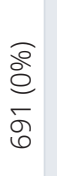 & 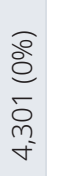 & $\begin{array}{l}\sqrt{0} \\
0 \\
\infty \\
\infty \\
\infty \\
\infty\end{array}$ \\
\hline 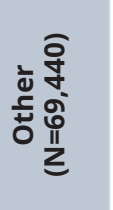 & 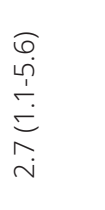 & $\begin{array}{l}\text { ô } \\
\text { î́ } \\
\text { d } \\
\text { y }\end{array}$ & & $\begin{array}{l}\bar{o} \\
\text { ò } \\
\text { n్d } \\
\text { o } \\
\text { o. } \\
m \\
m\end{array}$ & 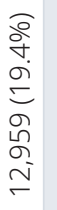 & 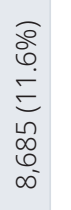 & 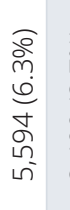 & $\begin{array}{l}\bar{a} \\
\hat{m} \\
\text { o } \\
\text { ò } \\
\dot{+} \\
m\end{array}$ & & 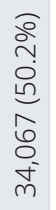 & 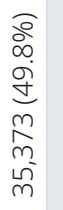 & & 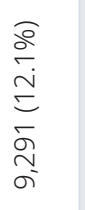 & 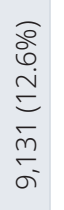 & 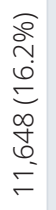 & 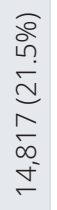 & \begin{tabular}{l}
$\widehat{o}$ \\
$b$ \\
$o$ \\
$\hat{n}$ \\
$\stackrel{n}{n}$ \\
\multirow{J}{*}{} \\
$\stackrel{\sim}{\sim}$
\end{tabular} & & 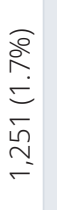 & 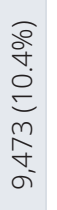 & 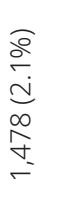 \\
\hline 总 & 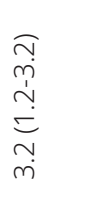 & $\begin{array}{l}\frac{a}{g} \\
\text { ó } \\
\text { d } \\
\text { J }\end{array}$ & & 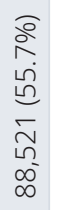 & 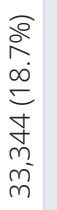 & 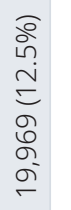 & $\begin{array}{l}\overline{0} \\
\stackrel{0}{\infty} \\
\infty \\
\circ \\
0 \\
0 \\
0 \\
-\end{array}$ & 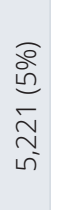 & & $\begin{array}{l}\widehat{a} \\
\frac{\rho}{\sigma} \\
\stackrel{d}{d} \\
\stackrel{n}{n} \\
\infty \\
\bar{\infty}\end{array}$ & 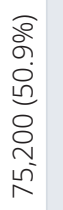 & & 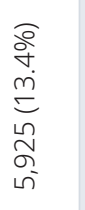 & 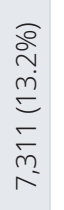 & 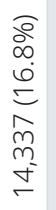 & 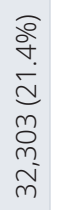 & 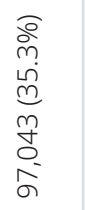 & & $\begin{array}{l}\widehat{o} \\
\infty \\
= \\
o \\
o \\
m\end{array}$ & 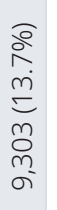 & 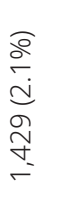 \\
\hline 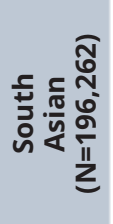 & 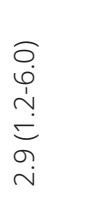 & 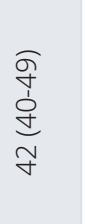 & & 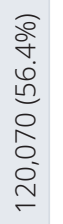 & 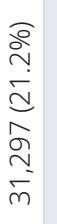 & 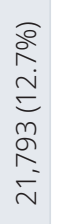 & 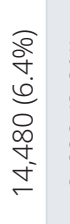 & 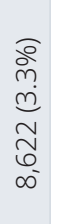 & & 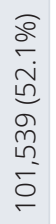 & 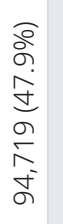 & & 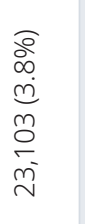 & 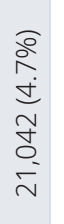 & 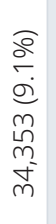 & 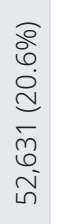 & 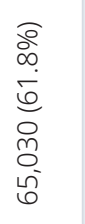 & & 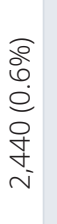 & $\begin{array}{l}\bar{a} \\
\text { ò } \\
\text { n. } \\
\text { o } \\
\infty \\
\infty \\
\infty\end{array}$ & 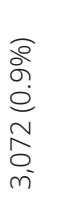 \\
\hline 点 & $\begin{array}{l}\infty 0 \\
\hat{0} \\
\dot{b} \\
\dot{E} \\
0 \\
\dot{+}\end{array}$ & 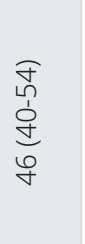 & & 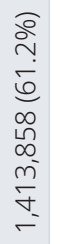 & 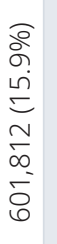 & 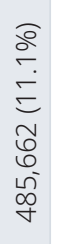 & 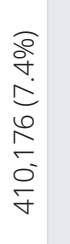 & 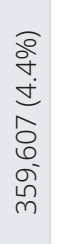 & & 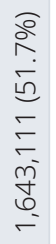 & 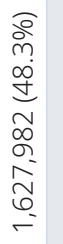 & & 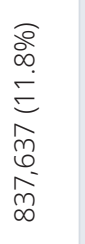 & 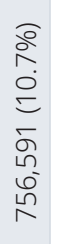 & 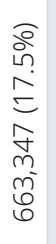 & 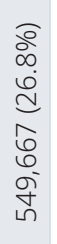 & 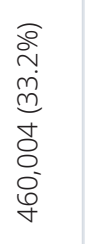 & & 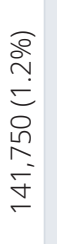 & 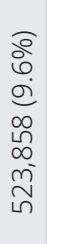 & 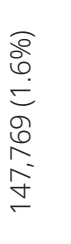 \\
\hline 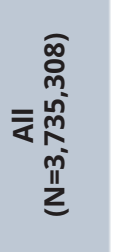 & 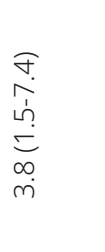 & 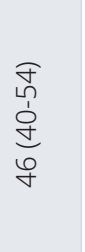 & & 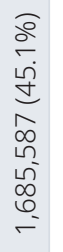 & 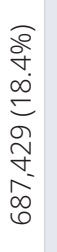 & 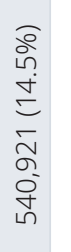 & 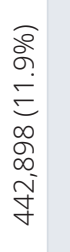 & 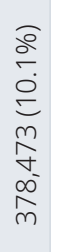 & & 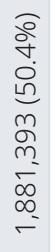 & 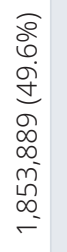 & & 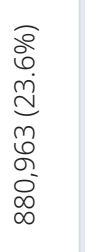 & 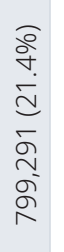 & 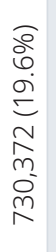 & 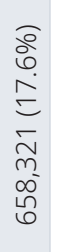 & 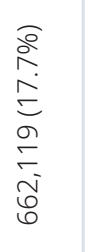 & & 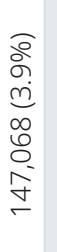 & 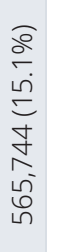 & 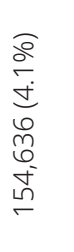 \\
\hline & 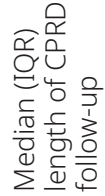 & 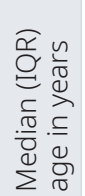 & 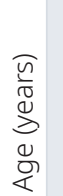 & 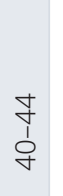 & $\begin{array}{l}\stackrel{g}{1} \\
\text { 岁 }\end{array}$ & 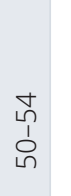 & 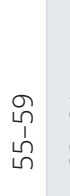 & $\begin{array}{l}0 \\
0 \\
1 \\
8\end{array}$ & 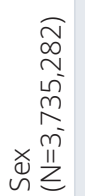 & $\frac{\frac{\sigma}{\pi}}{\sum}$ & 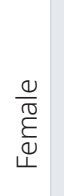 & 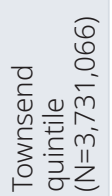 & 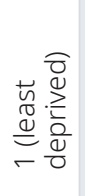 & $\sim$ & $m$ & $\nabla$ & 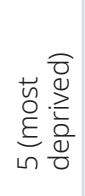 & 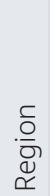 & 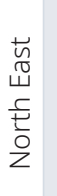 & 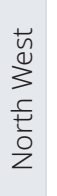 & 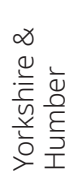 \\
\hline
\end{tabular}




\begin{tabular}{|c|c|c|c|c|c|c|c|c|c|c|c|c|c|c|c|c|c|c|c|c|}
\hline 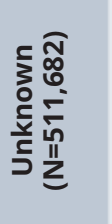 & 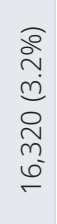 & 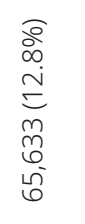 & 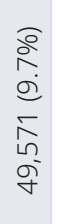 & 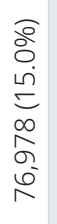 & 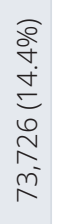 & 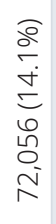 & $\begin{array}{l}\text { ò } \\
\infty \\
\bar{c} \\
\bar{c} \\
0 \\
0 \\
0 \\
0 \\
0\end{array}$ & $\begin{array}{l}\text { ָ̦ } \\
\text { อ̃ } \\
0\end{array}$ & & 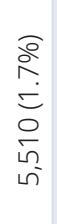 & 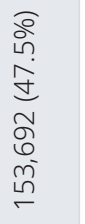 & 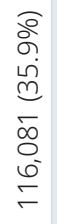 & 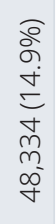 & & 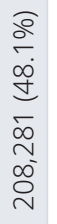 & 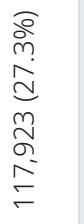 & $\begin{array}{l}\widehat{o} \\
b \\
\stackrel{+}{d} \\
m \\
0 \\
m \\
0 \\
0 \\
0\end{array}$ & & $\begin{array}{l}\bar{o} \\
80 \\
o \\
o \\
N \\
N \\
0 \\
0 \\
m \\
m\end{array}$ & 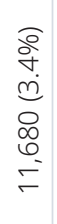 \\
\hline 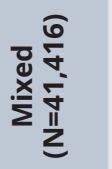 & $\begin{array}{l}0 \\
\stackrel{0}{0} \\
0 \\
0 \\
1\end{array}$ & $\begin{array}{l}\widehat{o} \\
\stackrel{0}{0} \\
\text { ñ } \\
\text { f }\end{array}$ & 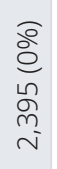 & $\begin{array}{l}\widehat{o} \\
o \\
o \\
m \\
\infty \\
\text { mi }\end{array}$ & 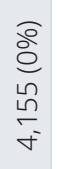 & $\begin{array}{l}\widehat{o} \\
0 \\
\infty \\
0 \\
0 \\
0 \\
0\end{array}$ & 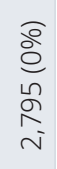 & 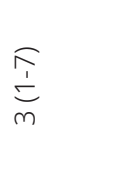 & & 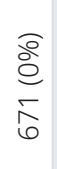 & 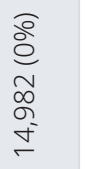 & 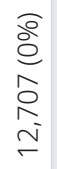 & 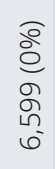 & & $\begin{array}{l}\bar{o} \\
\frac{0}{0} \\
\frac{1}{b} \\
\infty^{-}\end{array}$ & \begin{tabular}{l}
$\widehat{o}$ \\
$\stackrel{0}{c}$ \\
\multirow{2}{*}{} \\
$\infty$ \\
0 \\
$\stackrel{0}{-}$
\end{tabular} & $\begin{array}{l}\bar{\varrho} \\
\stackrel{0}{\infty} \\
\stackrel{\infty}{m} \\
\stackrel{+}{=}\end{array}$ & & 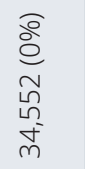 & $\begin{array}{l}\text { ò } \\
\text { o } \\
\text { o } \\
\text { ह- }\end{array}$ \\
\hline 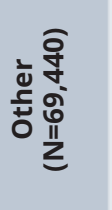 & 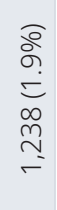 & 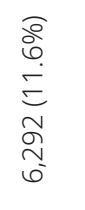 & 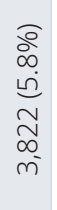 & 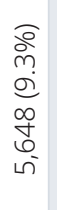 & 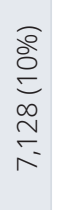 & 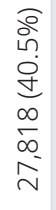 & 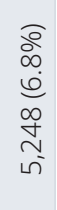 & $\begin{array}{l}\widehat{Q} \\
\stackrel{d}{v} \\
\sim\end{array}$ & & 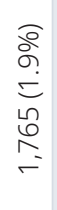 & 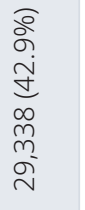 & 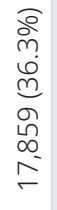 & 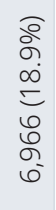 & & 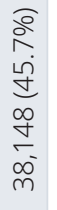 & $\begin{array}{l}\widehat{a} \\
\dot{o} \\
\dot{b} \\
\stackrel{d}{ } \\
o \\
\infty \\
o \\
m \\
\text { m. }\end{array}$ & 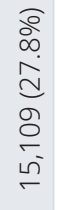 & & 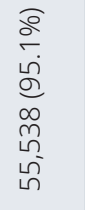 & 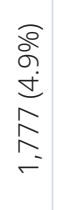 \\
\hline 旁 & 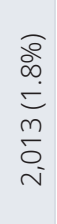 & 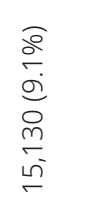 & $\begin{array}{l}\bar{o} \\
\text { in } \\
\text { மn } \\
\text { ᄋे } \\
\text { m. } \\
\text { ñ }\end{array}$ & $\begin{array}{l}\bar{a} \\
\stackrel{\infty}{\infty} \\
\infty \\
\infty \\
\infty \\
\sim \\
\infty\end{array}$ & 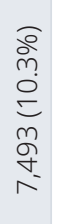 & 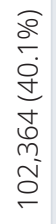 & $\begin{array}{l}\bar{\sigma} \\
b \\
\vdots \\
\bar{\sigma} \\
\bar{\sigma} \\
\sigma\end{array}$ & 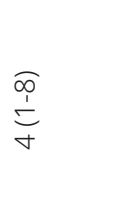 & & 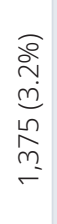 & 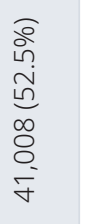 & 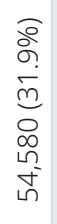 & 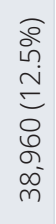 & & 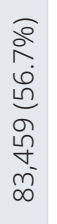 & 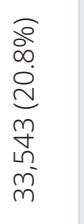 & 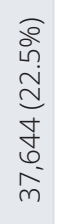 & & $\begin{array}{l}\bar{o} \\
o \\
b \\
o \\
0 \\
0 \\
\infty \\
\text { m. } \\
m\end{array}$ & 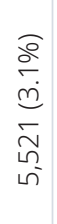 \\
\hline 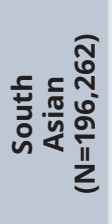 & 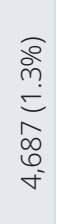 & 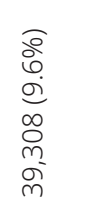 & 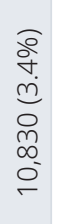 & 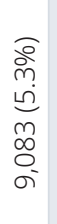 & $\begin{array}{l}\bar{o} \\
\infty \\
\dot{d} \\
o \\
o \\
o \\
\infty \\
\infty\end{array}$ & 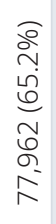 & 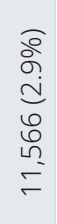 & 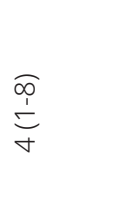 & & $\begin{array}{l}\bar{a} \\
\stackrel{5}{o} \\
\infty \\
\infty \\
o \\
\text { m }\end{array}$ & 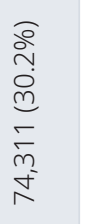 & 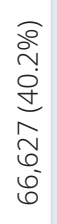 & 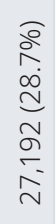 & & 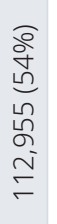 & 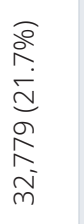 & 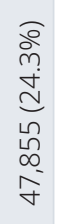 & & 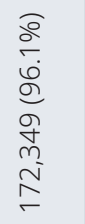 & 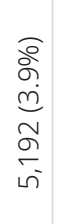 \\
\hline 点 & 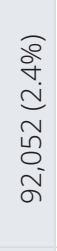 & 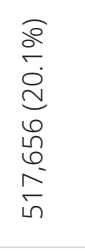 & 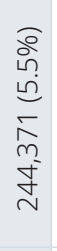 & 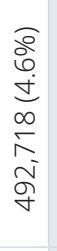 & 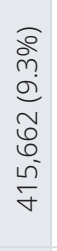 & 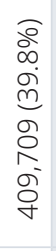 & 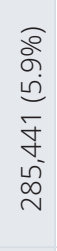 & $\underset{\substack{I \\
m}}{\stackrel{I}{I}}$ & & 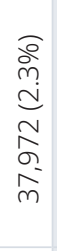 & 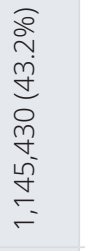 & 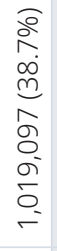 & 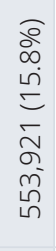 & & 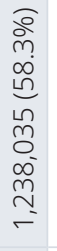 & 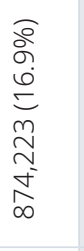 & 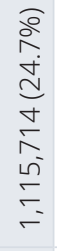 & & 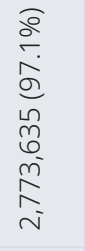 & 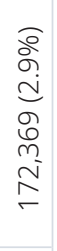 \\
\hline \multirow[t]{2}{*}{ 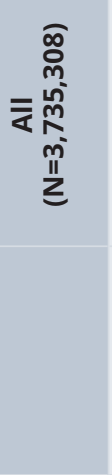 } & 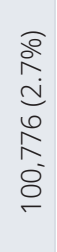 & 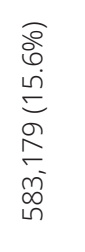 & 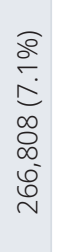 & 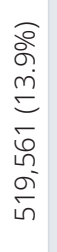 & 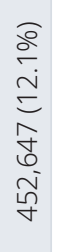 & 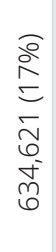 & 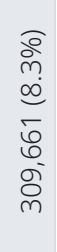 & 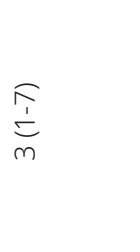 & & 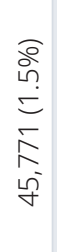 & 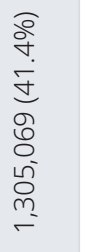 & 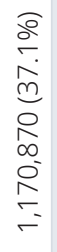 & 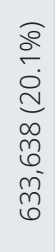 & & 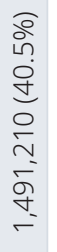 & 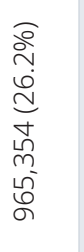 & 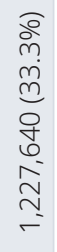 & & 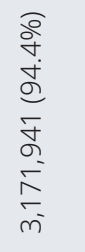 & $\begin{array}{l}\bar{o} \\
0 \\
0 \\
0 \\
0 \\
0 \\
0 \\
0 \\
\infty \\
\infty\end{array}$ \\
\hline & 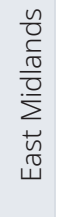 & 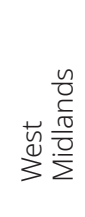 & 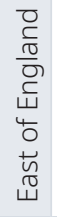 & 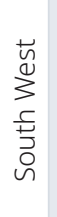 & 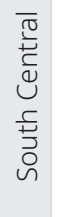 & 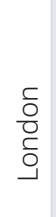 & 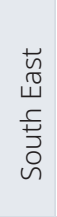 & 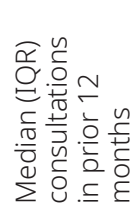 & 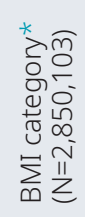 & $\begin{array}{l}\frac{\overline{0}}{0} \\
\frac{3}{0} \\
\frac{0}{0} \\
\text { 5 }\end{array}$ & 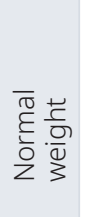 & $\begin{array}{l}\frac{7}{0} \\
\frac{0}{9} \\
\sum_{0}^{0} \\
0 \\
0\end{array}$ & $\begin{array}{l}\ddot{凶} \\
\stackrel{0}{0} \\
\text { On }\end{array}$ & 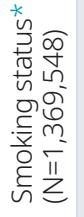 & 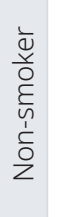 & 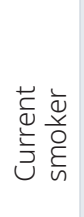 & 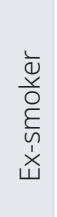 & 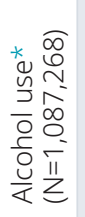 & 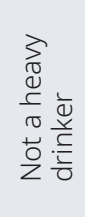 & 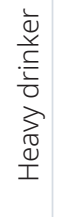 \\
\hline
\end{tabular}




\begin{tabular}{|c|c|c|c|c|c|c|c|c|c|c|c|c|c|c|c|c|c|c|c|c|c|}
\hline 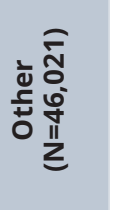 & 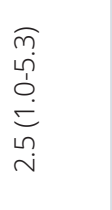 & 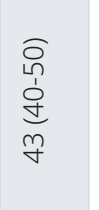 & & 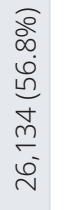 & 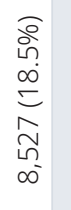 & 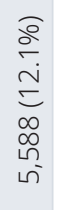 & 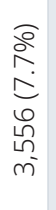 & 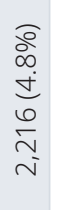 & & 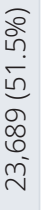 & 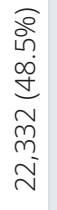 & & 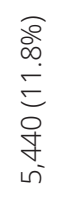 & 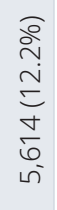 & $\begin{array}{l}\bar{o} \\
b \\
o \\
b \\
\stackrel{n}{n}\end{array}$ & 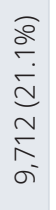 & 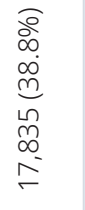 & & $\begin{array}{l}\text { ò } \\
\stackrel{1}{=} \\
\text { ô } \\
\sigma\end{array}$ & 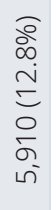 & 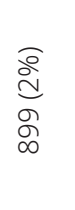 \\
\hline 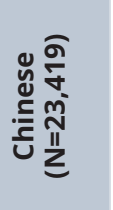 & 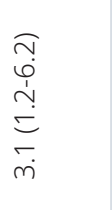 & $\begin{array}{l}\text { in } \\
\dot{d} \\
\dot{y}\end{array}$ & & 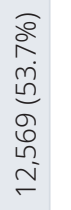 & 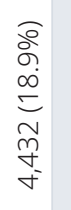 & 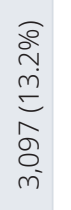 & $\begin{array}{l}\bar{o} \\
\grave{1} \\
\infty \\
\infty \\
0 \\
0 \\
i\end{array}$ & 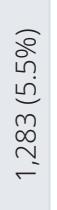 & & 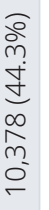 & 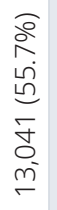 & & 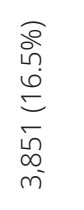 & 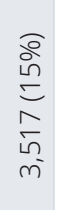 & 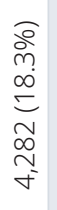 & 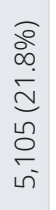 & 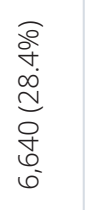 & & $\begin{array}{l}\widehat{a} \\
\stackrel{d}{d} \\
\stackrel{d}{\infty} \\
\stackrel{0}{n}\end{array}$ & 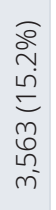 & 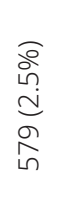 \\
\hline 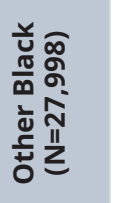 & 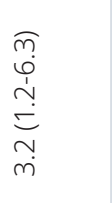 & 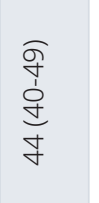 & & $\begin{array}{l}\bar{o} \\
\stackrel{0}{.} \\
\hat{\omega} \\
o \\
o \\
o \\
0 \\
0\end{array}$ & 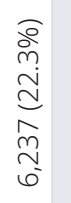 & 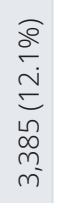 & 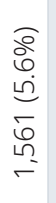 & 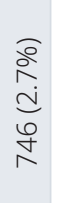 & & 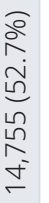 & 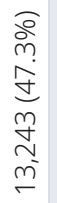 & & $\begin{array}{l}\bar{a} \\
\infty \\
\stackrel{+}{ \pm} \\
\widehat{m} \\
\stackrel{m}{-}\end{array}$ & 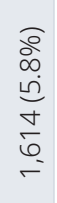 & 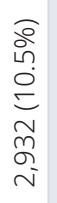 & 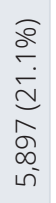 & 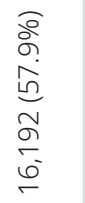 & & $\begin{array}{l}\widehat{a} \\
\stackrel{a}{1} \\
0 \\
\qquad 0 \\
\infty \\
\infty\end{array}$ & 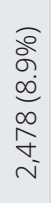 & $\begin{array}{l}\stackrel{a}{a} \\
\stackrel{N}{\infty} \\
\stackrel{\infty}{N}\end{array}$ \\
\hline 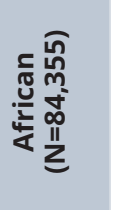 & 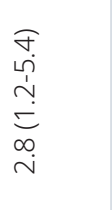 & 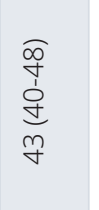 & & 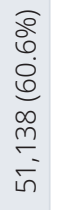 & 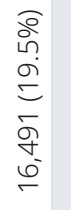 & 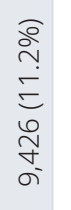 & 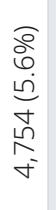 & 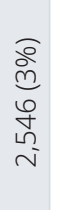 & & 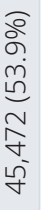 & $\begin{array}{l}\bar{o} \\
\dot{b} \\
\dot{b} \\
m \\
\infty \\
\infty \\
\infty \\
m\end{array}$ & & 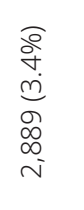 & 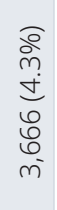 & 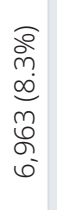 & 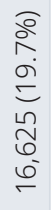 & 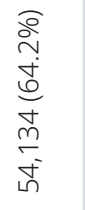 & & $\begin{array}{l}\bar{a} \\
\infty \\
0 \\
0 \\
\infty \\
0\end{array}$ & 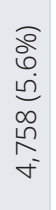 & $\begin{array}{l}\bar{\Phi} \\
\stackrel{5}{\circ} \\
\text { oे } \\
\text { के }\end{array}$ \\
\hline 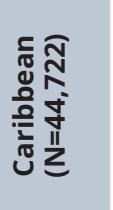 & 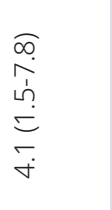 & 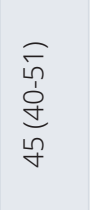 & & 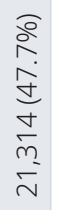 & 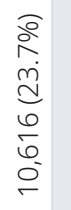 & $\begin{array}{l}\widehat{a} \\
0 \\
\stackrel{0}{c} \\
\infty \\
\stackrel{n}{r} \\
=\end{array}$ & $\begin{array}{l}\bar{a} \\
m \\
\infty \\
\infty \\
n \\
م \\
m \\
m\end{array}$ & 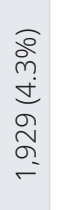 & & 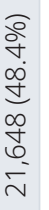 & 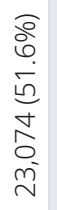 & & 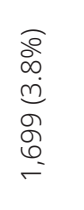 & 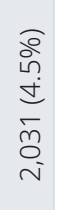 & 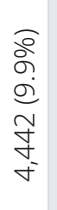 & 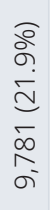 & 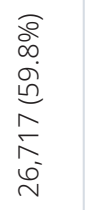 & & 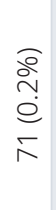 & $\begin{array}{l}\bar{o} \\
\dot{b} \\
\dot{j} \\
\hat{b} \\
0 \\
i\end{array}$ & 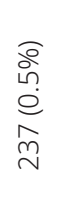 \\
\hline 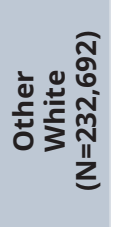 & 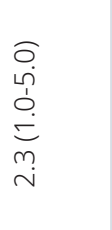 & $\begin{array}{l}\text { o } \\
\text { hn } \\
\text { ô } \\
\text { In } \\
\text { m }\end{array}$ & & 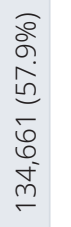 & 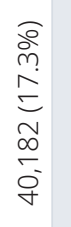 & 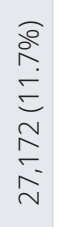 & $\begin{array}{l}0 \\
\infty \\
\infty \\
\infty \\
\infty \\
0 \\
\infty \\
\infty\end{array}$ & 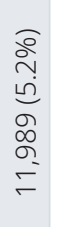 & & 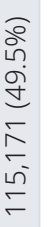 & 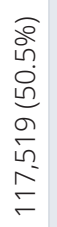 & & 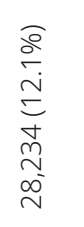 & 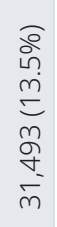 & 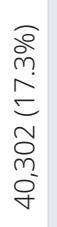 & 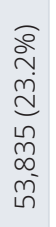 & $\begin{array}{l}\widehat{o} \\
\infty \\
m \\
m \\
0 \\
0 \\
\stackrel{0}{0} \\
\infty \\
\infty\end{array}$ & & 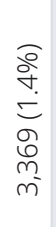 & $\begin{array}{l}\bar{o} \\
o \\
\text { ô } \\
\circ \\
0 \\
0 \\
0 \\
0\end{array}$ & 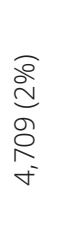 \\
\hline 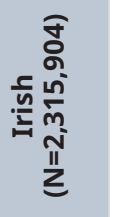 & 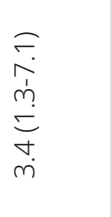 & 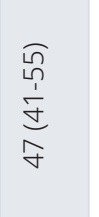 & & 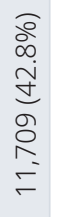 & 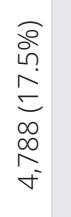 & 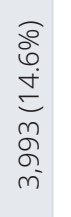 & 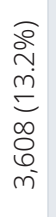 & 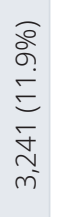 & & 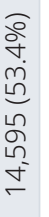 & 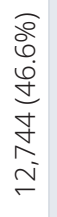 & & 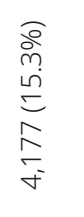 & 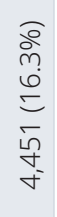 & 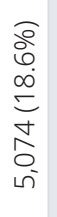 & 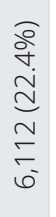 & 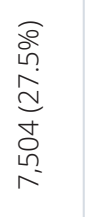 & & 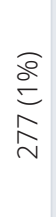 & 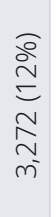 & $\begin{array}{l}\text { ò } \\
\text { in } \\
\stackrel{5}{\vdots} \\
\text { f }\end{array}$ \\
\hline 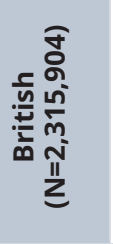 & 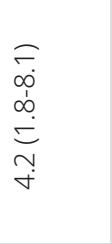 & 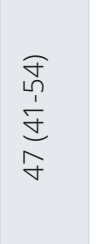 & & 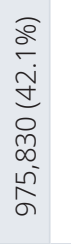 & 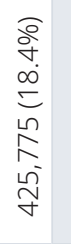 & 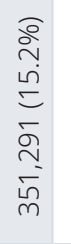 & 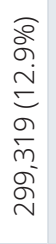 & 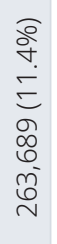 & & 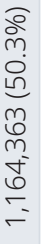 & 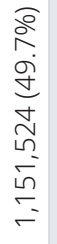 & & 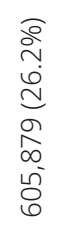 & 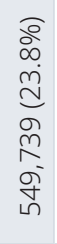 & 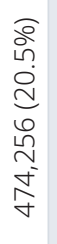 & 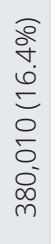 & 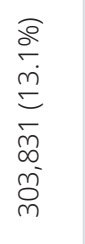 & & 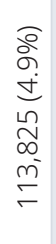 & $\begin{array}{l}\widehat{o} \\
\infty \\
0 \\
\varrho \\
\infty \\
\infty \\
\sigma \\
\sigma \\
\infty \\
\infty \\
m\end{array}$ & 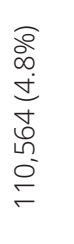 \\
\hline & 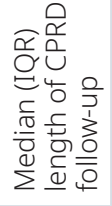 & 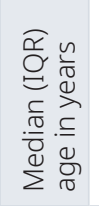 & 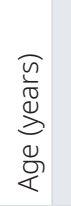 & $\begin{array}{l}\text { \& } \\
1 \\
\text { o }\end{array}$ & 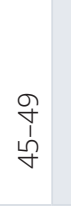 & $\begin{array}{l}\text { t. } \\
\text { 1 } \\
\text { in }\end{array}$ & $\begin{array}{l}\text { 员 } \\
\text { 占 } \\
\text { nn }\end{array}$ & 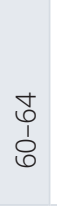 & $\stackrel{\times}{ٌ}$ & $\sum^{\frac{\pi}{\pi}}$ & 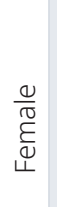 & 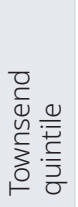 & 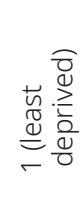 & $\sim$ & $m$ & $\nabla$ & 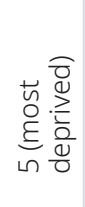 & $\begin{array}{l}\ulcorner \\
\frac{.}{\sigma} \\
\simeq \\
\simeq\end{array}$ & 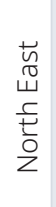 & 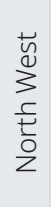 & 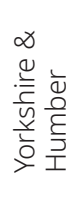 \\
\hline
\end{tabular}




\begin{tabular}{|c|c|c|c|c|c|c|c|c|c|c|c|c|c|c|c|c|c|c|c|c|}
\hline 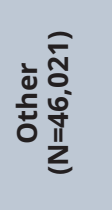 & 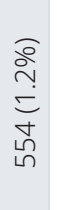 & $\begin{array}{l}a \\
\infty \\
\infty \\
\infty \\
0 \\
0 \\
m\end{array}$ & 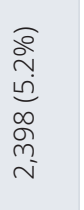 & $\begin{array}{l}\frac{a}{a} \\
\frac{0}{\infty} \\
\hat{\infty} \\
\hat{2} \\
m\end{array}$ & $\begin{array}{l}\bar{o} \\
8 \\
5 \\
0 \\
\infty \\
\stackrel{0}{+} \\
+\end{array}$ & 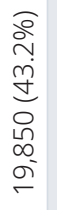 & 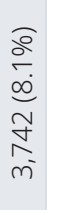 & $\begin{array}{l}\underset{f}{\rho} \\
\text { d }\end{array}$ & & 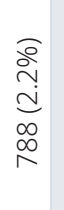 & 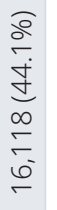 & 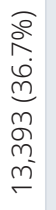 & 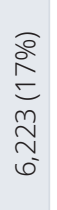 & & 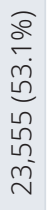 & 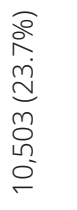 & $\begin{array}{l}\text { ò } \\
\text { ñ. } \\
\text { ḋ } \\
\text { o } \\
\text { m. } \\
\text { o }\end{array}$ & & 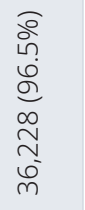 & $\begin{array}{l}\text { ò } \\
i n \\
\stackrel{n}{n} \\
\infty \\
\text { m. } \\
\text { ñ }\end{array}$ \\
\hline 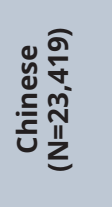 & 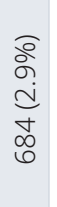 & 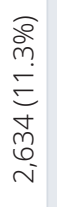 & 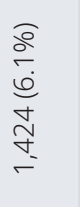 & 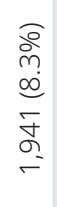 & 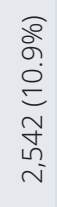 & 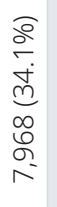 & 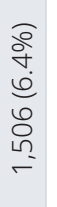 & $\begin{array}{l}\text { ఢn } \\
\stackrel{\rho}{\sim} \\
\sim\end{array}$ & & $\begin{array}{l}\text { io } \\
\text { in } \\
\text { 命 }\end{array}$ & 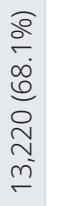 & 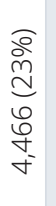 & $\begin{array}{l}\bar{o} \\
\infty \\
m \\
m \\
\stackrel{N}{N}\end{array}$ & & 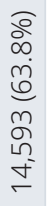 & 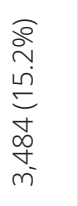 & 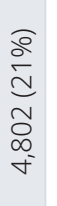 & & 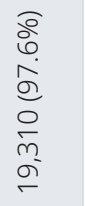 & 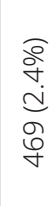 \\
\hline 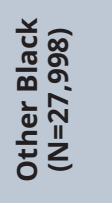 & 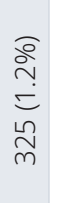 & 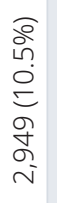 & 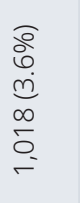 & 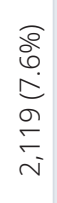 & 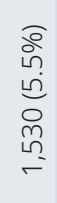 & 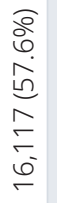 & $\begin{array}{l}\bar{o} \\
\text { iñ } \\
\text { ñ } \\
\text { o. } \\
\text { aे }\end{array}$ & 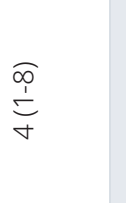 & & 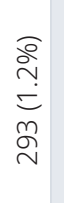 & 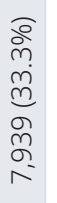 & 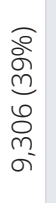 & 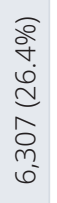 & & 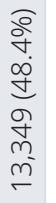 & 高 & 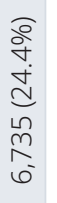 & & 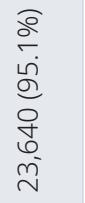 & 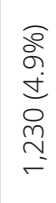 \\
\hline 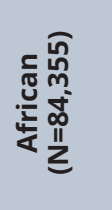 & 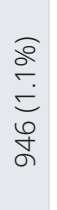 & 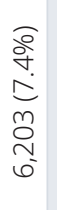 & $\begin{array}{l}\bar{o} \\
\text { ò } \\
\text { mं } \\
\text { ò } \\
\text { ò } \\
\text { i }\end{array}$ & 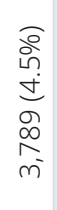 & 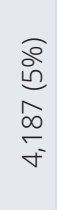 & 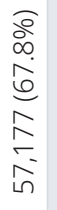 & 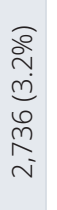 & $\begin{array}{l}\underset{\infty}{\stackrel{\infty}{c}} \\
m\end{array}$ & & $\begin{array}{l}\overline{0} \\
\text { ò } \\
\stackrel{0}{1} \\
\tilde{n} \\
0\end{array}$ & 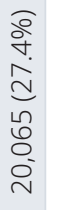 & 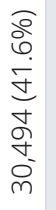 & 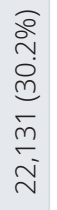 & & 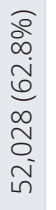 & 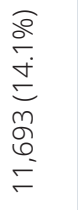 & 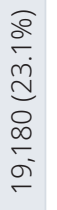 & & $\begin{array}{l}\bar{a} \\
\hat{0} \\
o \\
o \\
\overline{0} \\
0 \\
\text { m }\end{array}$ & 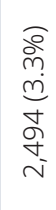 \\
\hline 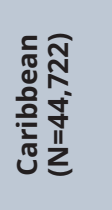 & 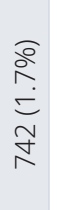 & 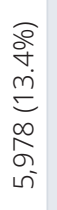 & $\begin{array}{l}\bar{o} \\
m \\
m \\
\infty \\
\infty \\
o \\
⿱ 亠 䒑 \\
r\end{array}$ & 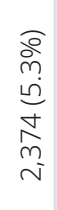 & $\begin{array}{l}\widehat{o} \\
\dot{d} \\
6 \\
\stackrel{0}{E} \\
=\end{array}$ & 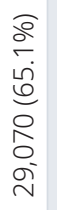 & 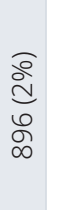 & $\begin{array}{l}\underset{\infty}{\infty} \\
\stackrel{\perp}{\forall} \\
\forall\end{array}$ & & $\begin{array}{l}\text { ò } \\
\text { N̦} \\
\stackrel{0}{0} \\
\text { bे }\end{array}$ & 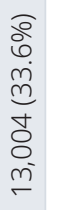 & 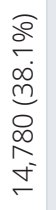 & 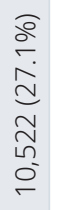 & & 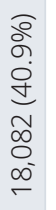 & 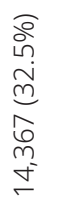 & 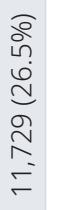 & & 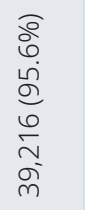 & $\begin{array}{l}\widehat{o} \\
\stackrel{8}{+} \\
\text { o } \\
\text { o } \\
\stackrel{-}{=}\end{array}$ \\
\hline 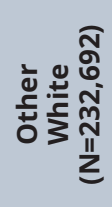 & 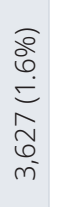 & 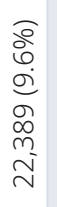 & 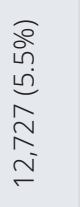 & 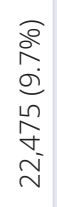 & 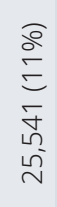 & 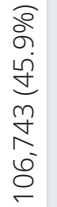 & 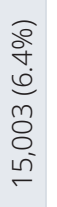 & 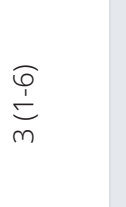 & & 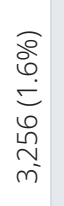 & 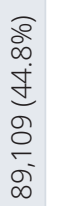 & 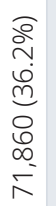 & 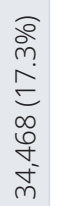 & & 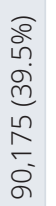 & 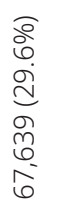 & 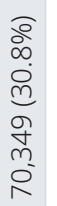 & & 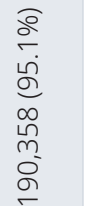 & 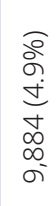 \\
\hline 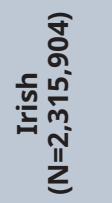 & 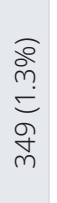 & $\begin{array}{l}\bar{a} \\
b \\
\dot{a} \\
\bar{\Sigma} \\
\bar{\Sigma} \\
\frac{1}{m}\end{array}$ & 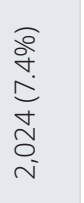 & 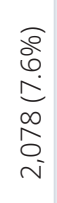 & $\begin{array}{l}\bar{a} \\
2 \\
\infty \\
\hat{\infty} \\
\hat{n} \\
i \\
v\end{array}$ & 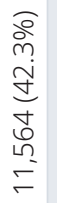 & 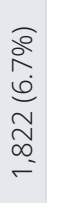 & 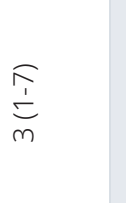 & & 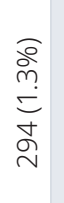 & 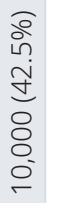 & $\begin{array}{l}\bar{\alpha} \\
\infty \\
\hat{\infty} \\
m \\
\infty \\
\infty \\
\infty \\
\infty \\
\infty\end{array}$ & 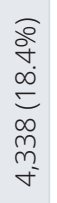 & & 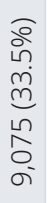 & 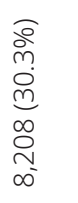 & 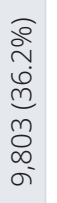 & & 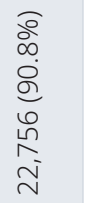 & 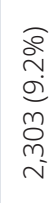 \\
\hline \multirow[t]{2}{*}{ 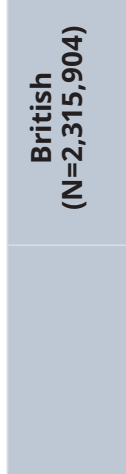 } & 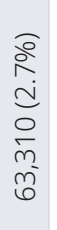 & 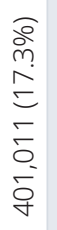 & 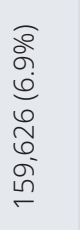 & 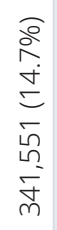 & 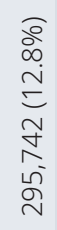 & 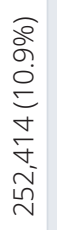 & 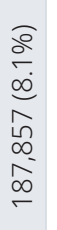 & $\frac{\underset{1}{\stackrel{1}{5}}}{m}$ & & 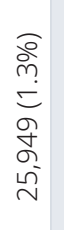 & 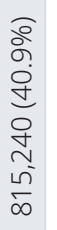 & 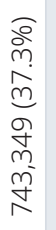 & 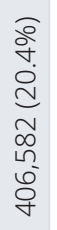 & & 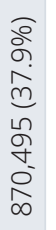 & 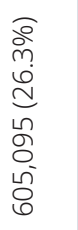 & 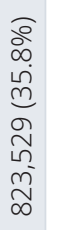 & & 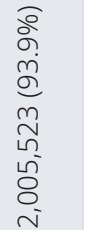 & 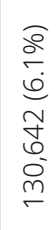 \\
\hline & 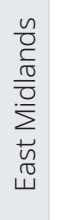 & 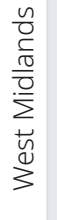 & 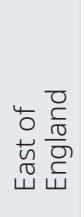 & 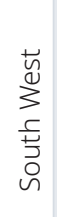 & 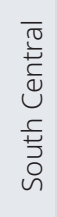 & 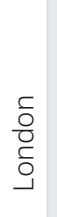 & 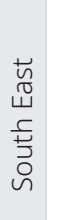 & 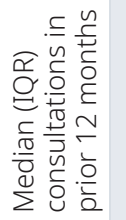 & 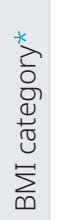 & $\begin{array}{l}\frac{ \pm}{0} \\
\frac{0}{0} \\
\frac{\sum_{0}}{0} \\
\frac{0}{5} \\
\frac{5}{5}\end{array}$ & 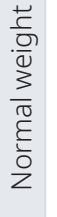 & $\begin{array}{l}\frac{.}{0} \\
\frac{.0}{0} \\
\frac{2}{0} \\
0 \\
0\end{array}$ & $\begin{array}{l}\stackrel{凶}{ } \\
\stackrel{0}{0}\end{array}$ & 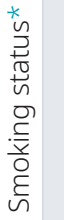 & 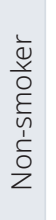 & 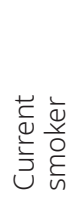 & 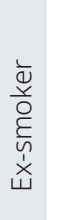 & 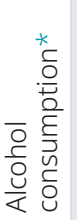 & 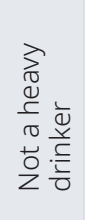 & 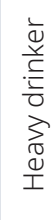 \\
\hline
\end{tabular}




\begin{tabular}{|c|c|c|c|c|c|c|c|c|c|c|c|c|c|c|c|c|c|c|c|c|c|c|c|}
\hline 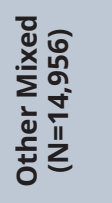 & 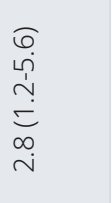 & 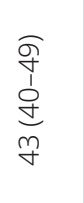 & & $\begin{array}{l}\widehat{o} \\
o \\
\infty \\
\infty \\
0 \\
\sigma \\
\infty \\
\infty \\
\infty\end{array}$ & $\begin{array}{l}0 \\
\infty \\
\infty \\
\infty \\
\infty \\
\infty \\
\infty \\
\infty \\
i\end{array}$ & 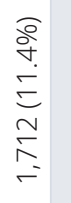 & 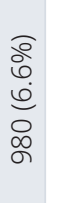 & 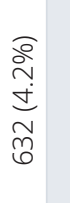 & 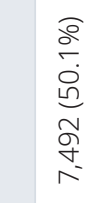 & 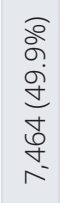 & & 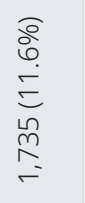 & 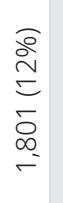 & 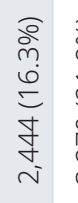 & 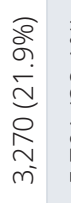 & 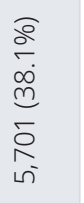 & & 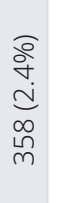 & 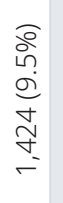 & $\begin{array}{l}\underset{o}{o} \\
\infty \\
\stackrel{\infty}{\leftrightarrows} \\
\stackrel{\sim}{v}\end{array}$ & 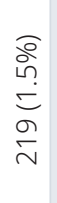 & 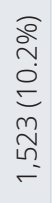 & 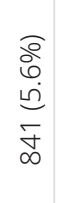 \\
\hline 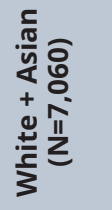 & 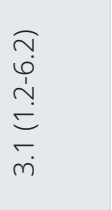 & 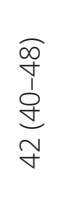 & & 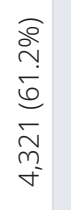 & 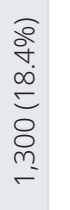 & 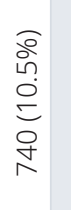 & $\begin{array}{l}\overline{0} \\
\text { in } \\
\text { é } \\
\bar{\sigma} \\
\text { q }\end{array}$ & 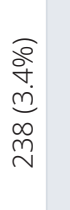 & 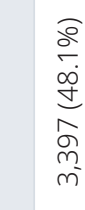 & 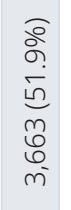 & & 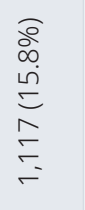 & 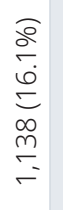 & 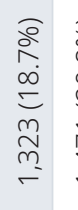 & 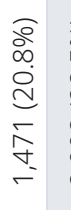 & 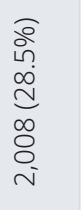 & & 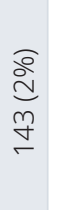 & 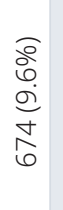 & 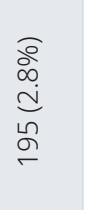 & 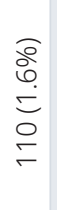 & 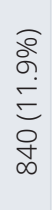 & $\begin{array}{l}\stackrel{\curvearrowright}{a} \\
\stackrel{m}{n} \\
\stackrel{m}{m} \\
m\end{array}$ \\
\hline 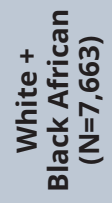 & 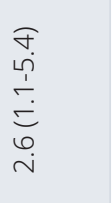 & $\begin{array}{l}\text { ơ } \\
\stackrel{+}{o} \\
\text { d } \\
\text { m }\end{array}$ & & 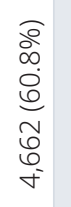 & 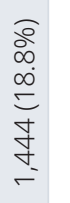 & 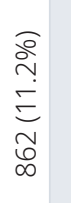 & $\begin{array}{l}\bar{o} \\
\dot{o} \\
\dot{\omega} \\
\tilde{y} \\
\tilde{y}\end{array}$ & 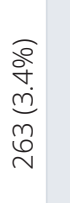 & $\begin{array}{l}\overline{0} \\
0 \\
\dot{0} \\
\stackrel{0}{0} \\
\infty \\
\infty \\
\mp\end{array}$ & 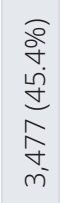 & & $\begin{array}{l}\bar{o} \\
\infty \\
\infty \\
\infty \\
\omega \\
\sigma\end{array}$ & 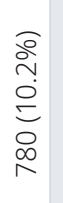 & 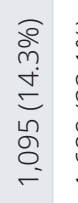 & 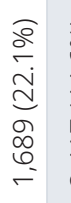 & 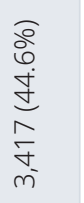 & & 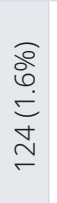 & 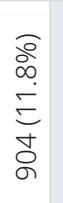 & 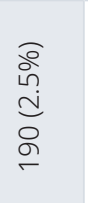 & 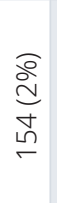 & 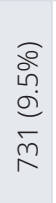 & $\begin{array}{l}\bar{d} \\
\text { ò } \\
\stackrel{+}{+} \\
\stackrel{+}{m}\end{array}$ \\
\hline 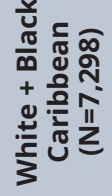 & $\begin{array}{l}\underset{N}{N} \\
\hat{m} \\
\stackrel{5}{j} \\
\dot{m} \\
m\end{array}$ & 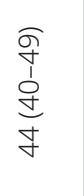 & & 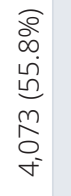 & 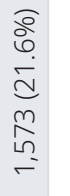 & 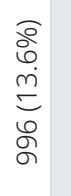 & 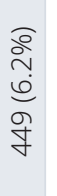 & 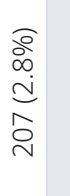 & $\begin{array}{l}\bar{a} \\
\stackrel{\circ}{\circ} \\
\text { in } \\
\infty \\
0 \\
0 \\
\text { m. } \\
\text { m. }\end{array}$ & $\begin{array}{l}\bar{o} \\
\text { ò } \\
\text { ò } \\
0 \\
0 \\
0 \\
0 \\
\text { m. }\end{array}$ & & 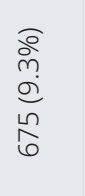 & 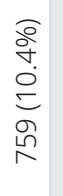 & 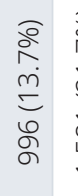 & 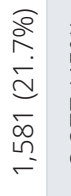 & 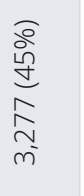 & & 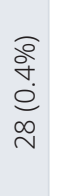 & 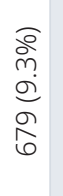 & $\begin{array}{l}\bar{o} \\
o \\
\stackrel{5}{=} \\
\hat{m}\end{array}$ & 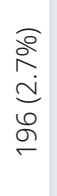 & 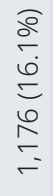 & 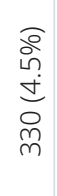 \\
\hline 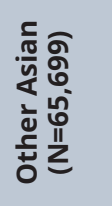 & 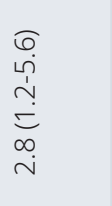 & 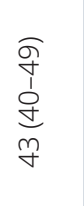 & & 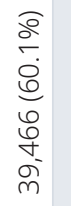 & 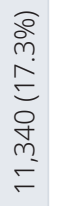 & 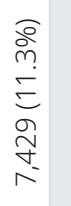 & 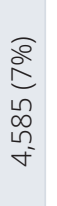 & 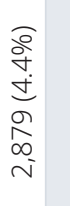 & 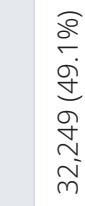 & 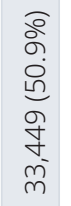 & & $\begin{array}{l}\bar{o} \\
o \\
0 \\
0 \\
0 \\
0 \\
\infty \\
0 \\
0\end{array}$ & 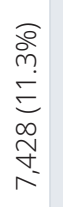 & 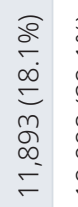 & 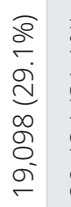 & 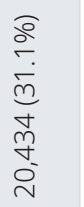 & & 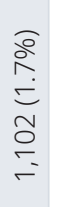 & 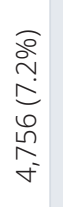 & 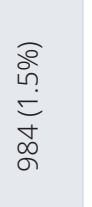 & & 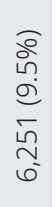 & 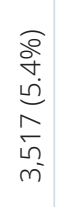 \\
\hline 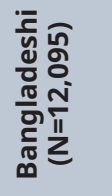 & 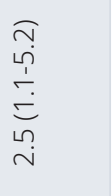 & 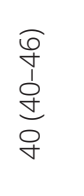 & & $\begin{array}{l}0 \\
o \\
0 \\
0 \\
\vdots \\
\infty \\
\infty \\
\infty \\
\infty\end{array}$ & 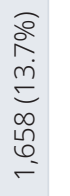 & 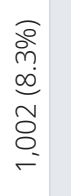 & 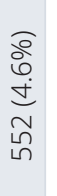 & 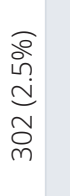 & 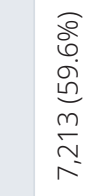 & 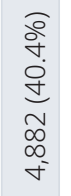 & & 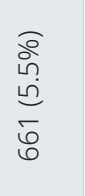 & 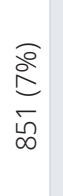 & 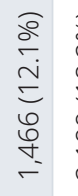 & 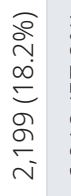 & 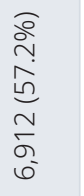 & & 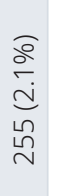 & 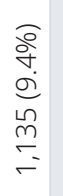 & 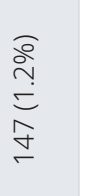 & 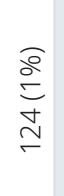 & $\begin{array}{l}\text { ò } \\
b \\
\stackrel{0}{\sigma} \\
m \\
o \\
\sigma\end{array}$ & $\begin{array}{l}\widehat{a} \\
i n \\
\infty \\
0 \\
0 \\
0 \\
\vdots\end{array}$ \\
\hline 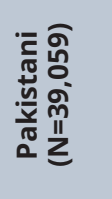 & 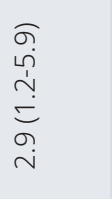 & 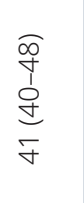 & & 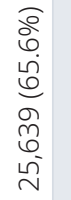 & 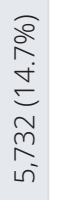 & $\begin{array}{l}\bar{o} \\
\stackrel{8}{ } \\
= \\
\bar{o} \\
\text { m }\end{array}$ & 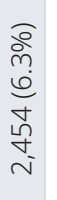 & 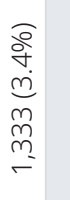 & 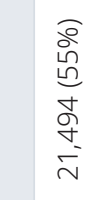 & 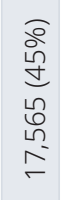 & & 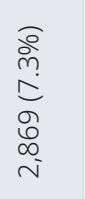 & 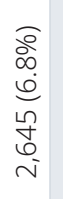 & 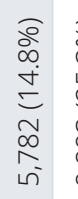 & 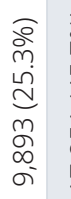 & 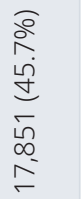 & & 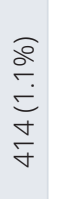 & 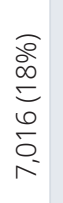 & $\begin{array}{l}\text { ò } \\
\text { dे } \\
\text { va } \\
\frac{n}{\sigma}\end{array}$ & 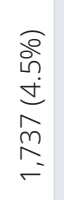 & 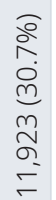 & 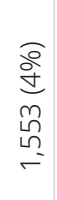 \\
\hline 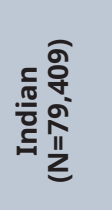 & 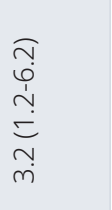 & $\begin{array}{l}\text { ồ } \\
\text { ô. } \\
\text { d } \\
\text { m }\end{array}$ & & 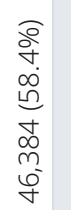 & 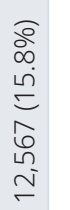 & $\begin{array}{l}\bar{o} \\
o \\
\bar{E} \\
\bar{\sigma} \\
\bar{\sigma} \\
\sigma \\
\sigma\end{array}$ & $\begin{array}{l}\overline{0} \\
\stackrel{0}{0} \\
\infty \\
\infty \\
\infty \\
\infty \\
0 \\
0\end{array}$ & 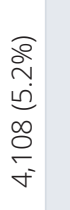 & 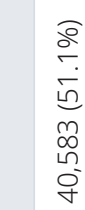 & $\begin{array}{l}\widehat{o} \\
o \\
\infty \\
\infty \\
\stackrel{\infty}{ \pm} \\
\underset{N}{ } \\
\infty \\
\infty \\
m\end{array}$ & & 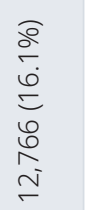 & 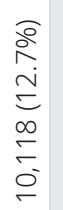 & 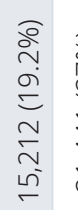 & $\begin{array}{l}\frac{a}{d} \\
\stackrel{d}{d} \\
\bar{j} \\
\bar{N}\end{array}$ & 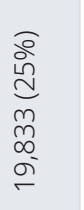 & & $\begin{array}{l}\bar{o} \\
\infty \\
o \\
o \\
o \\
b\end{array}$ & 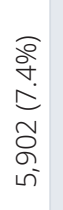 & 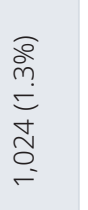 & 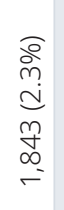 & 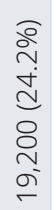 & 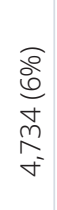 \\
\hline & 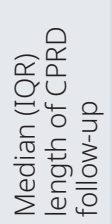 & 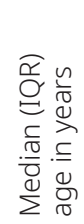 & 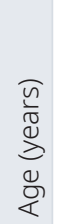 & $\begin{array}{l}\text { 寸 } \\
\text { 1 } \\
\text { o }\end{array}$ & 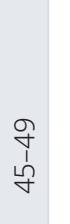 & 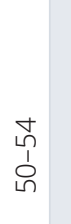 & 角 & $\begin{array}{l}0 \\
1\end{array}$ & 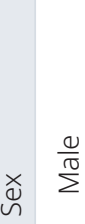 & $\begin{array}{l}\frac{\mathscr{U}}{\widetilde{\sigma}} \\
\stackrel{\varepsilon}{\Psi} \\
\Psi\end{array}$ & 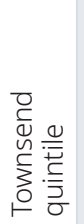 & 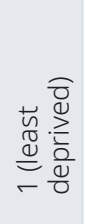 & N & & . & 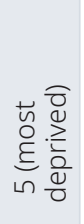 & 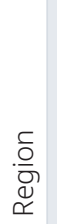 & 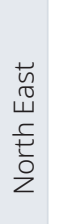 & 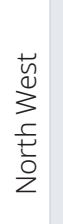 & 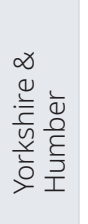 & 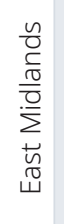 & 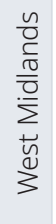 & 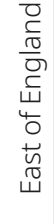 \\
\hline
\end{tabular}




\begin{tabular}{|c|c|c|c|c|c|c|c|c|c|c|c|c|c|c|c|c|c|}
\hline 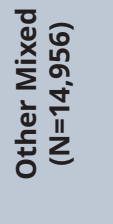 & 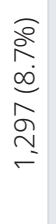 & $\begin{array}{l}\bar{o} \\
\infty \\
\infty \\
\infty \\
\stackrel{m}{m} \\
\stackrel{m}{r}\end{array}$ & 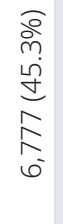 & $\begin{array}{l}\bar{\sigma} \\
\frac{\sigma}{\sigma} \\
\frac{0}{\sigma} \\
\sigma\end{array}$ & 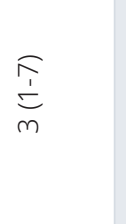 & & $\begin{array}{l}\underset{d}{d} \\
\stackrel{d}{d}\end{array}$ & 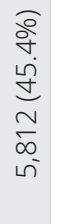 & 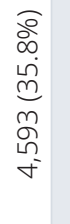 & 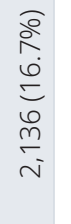 & & 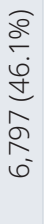 & 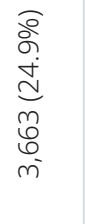 & 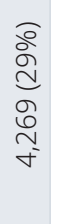 & & 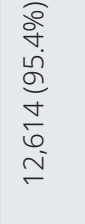 & $\begin{array}{l}\bar{o} \\
b \\
\dot{d} \\
\text { o } \\
b\end{array}$ \\
\hline $\begin{array}{l}\frac{5}{\frac{\pi}{3}} \\
\frac{1}{4} \\
+\frac{0}{0} \\
+\frac{\pi}{\pi} \\
\frac{\pi}{3} \\
\frac{11}{3}\end{array}$ & $\begin{array}{l}\widehat{o} \\
\frac{a}{\sigma} \\
\frac{m}{b}\end{array}$ & 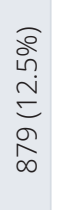 & 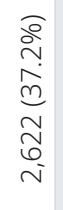 & 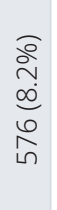 & $\underset{\substack{I \\
m}}{\stackrel{F}{I}}$ & & 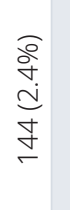 & 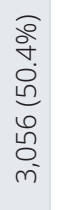 & $\begin{array}{l}\bar{a} \\
\dot{a} \\
\dot{j} \\
0 \\
o \\
0 \\
i \\
i\end{array}$ & 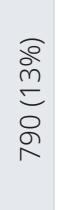 & & 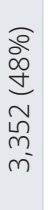 & 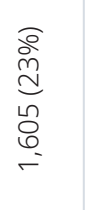 & 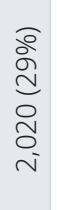 & & 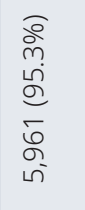 & 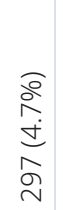 \\
\hline 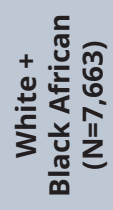 & 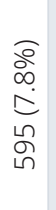 & 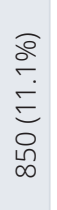 & 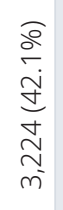 & 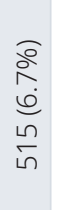 & $\underset{m}{\stackrel{i}{\leftrightarrows}}$ & & 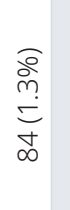 & $\begin{array}{l}\bar{\varrho} \\
\text { in } \\
m \\
m \\
o \\
o \\
\stackrel{i}{i}\end{array}$ & 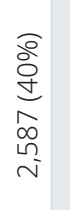 & 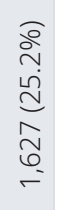 & & 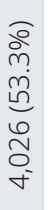 & 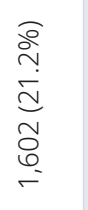 & 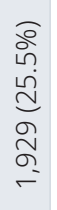 & & 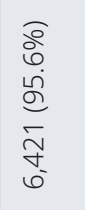 & 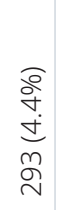 \\
\hline 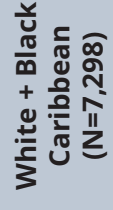 & 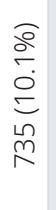 & 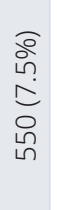 & 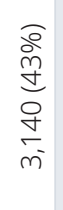 & 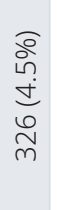 & 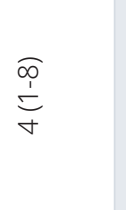 & & 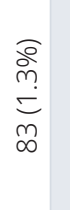 & 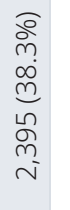 & $\begin{array}{l}\bar{a} \\
b \\
\hat{m} \\
m \\
m \\
m \\
m \\
i\end{array}$ & 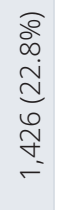 & & 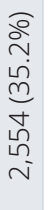 & 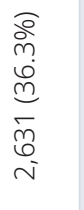 & $\begin{array}{l}\overline{0} \\
i \\
0 \\
\infty \\
\stackrel{0}{0} \\
o \\
o \\
o \\
i\end{array}$ & & 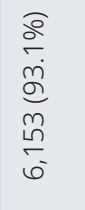 & 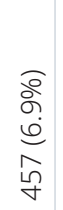 \\
\hline 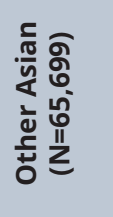 & 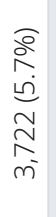 & $\begin{array}{l}\bar{o} \\
\infty \\
0 \\
0 \\
\bar{\sigma} \\
\sigma \\
\bar{n} \\
\end{array}$ & $\begin{array}{l}\widehat{a} \\
\infty \\
\infty \\
\dot{0} \\
\sim \\
0 \\
0 \\
\text { i } \\
m\end{array}$ & 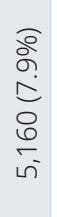 & 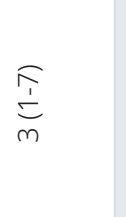 & & 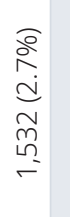 & 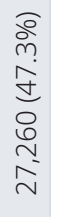 & $\begin{array}{l}\bar{a} \\
\infty \\
0 \\
m \\
m \\
m \\
\infty \\
\bar{c} \\
\bar{v}\end{array}$ & 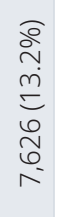 & & $\begin{array}{l}\bar{a} \\
o \\
\infty \\
\infty \\
\infty \\
\infty \\
0 \\
0 \\
\dot{m}\end{array}$ & $\begin{array}{l}\widehat{o} \\
\text { oे } \\
0 \\
气 \\
\circ \\
o \\
o \\
\circ \\
\circ\end{array}$ & 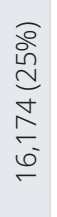 & & 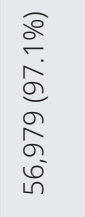 & $\begin{array}{l}\bar{a} \\
\text { àd } \\
\stackrel{0}{0} \\
\stackrel{N}{=}\end{array}$ \\
\hline 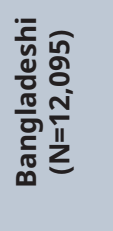 & 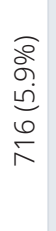 & 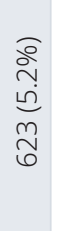 & 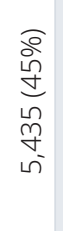 & $\begin{array}{l}\bar{o} \\
b \\
\dot{\omega} \\
\infty \\
0 \\
6\end{array}$ & 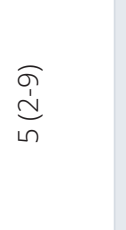 & & $\begin{array}{l}\bar{a} \\
\stackrel{i}{i} \\
\stackrel{0}{0} \\
\stackrel{v}{v}\end{array}$ & 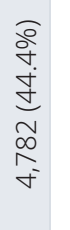 & 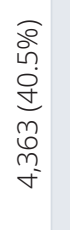 & 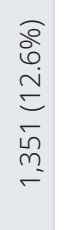 & & 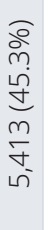 & 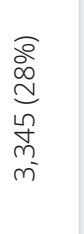 & 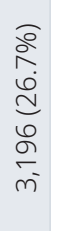 & & $\begin{array}{l}\bar{a} \\
\stackrel{0}{\hat{a}} \\
\hat{0} \\
\infty \\
0 \\
0 \\
0\end{array}$ & 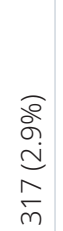 \\
\hline 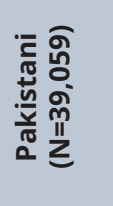 & 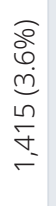 & 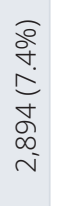 & 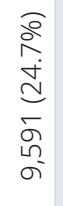 & 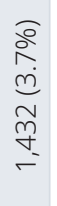 & $\underset{\forall}{\stackrel{\sigma}{r}}$ & & 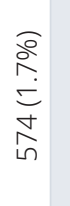 & 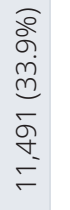 & 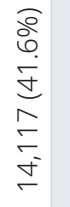 & 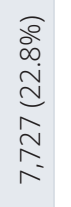 & & 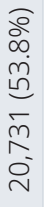 & 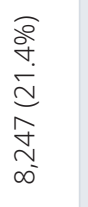 & $\begin{array}{l}\bar{o} \\
\infty \\
\stackrel{d}{d} \\
\bar{d} \\
\infty \\
\stackrel{\omega}{\sigma} \\
\sigma\end{array}$ & & $\begin{array}{l}\bar{o} \\
\infty \\
0 \\
o \\
0 \\
o \\
0 \\
\infty \\
\text { m. }\end{array}$ & 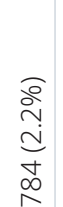 \\
\hline \multirow[t]{2}{*}{ 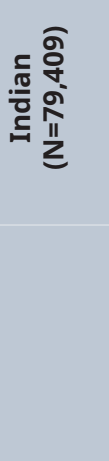 } & 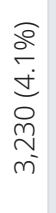 & $\begin{array}{l}\bar{o} \\
\stackrel{2}{20} \\
\stackrel{2}{m} \\
\stackrel{n}{n} \\
\end{array}$ & $\begin{array}{l}\bar{o} \\
o \\
\infty \\
0 \\
\infty \\
\infty \\
\infty \\
\infty \\
m\end{array}$ & 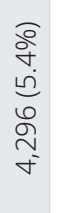 & $\underset{m}{\stackrel{ }{5}}$ & & 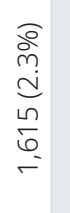 & 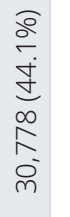 & 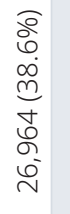 & 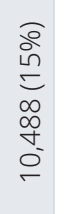 & & 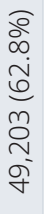 & 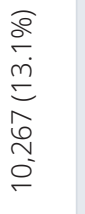 & 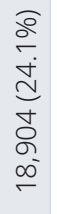 & & 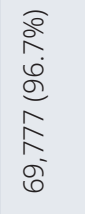 & 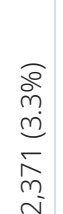 \\
\hline & 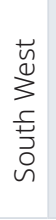 & 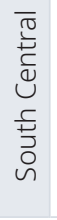 & $\begin{array}{l}\overline{0} \\
\overline{0} \\
\overline{0}\end{array}$ & 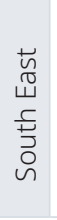 & 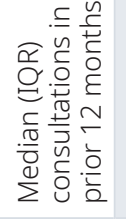 & 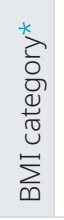 & 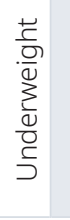 & 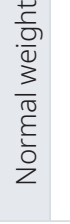 & 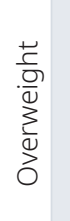 & 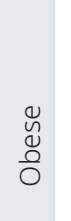 & 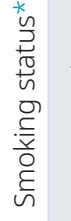 & 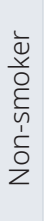 & 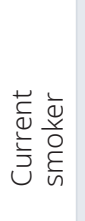 & 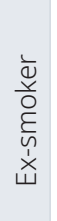 & 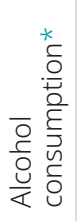 & 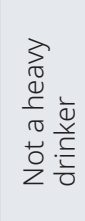 & 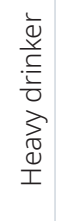 \\
\hline
\end{tabular}


We identified 102,316 influenza/ILI episodes recorded among 94,623 patients, and 560,860 ARI episodes among 421,349 patients. The rate of influenza/ILI was highest in the South Asian group (9.6 per 1,000 person-years) followed by the Black group (8.4 per 1,000 person-years) (Figure 2, Table 4). In all ethnic groups the influenza/ILI rates were higher in women than men and decreased with age.

After adjustment for age, sex and year, the incidence rate ratio (IRR) for influenza/ILI was higher for South Asian (1.70, 95\% CI 1.66-1.75), Black (1.48, 95\% CI 1.44-1.53), and Mixed (1.22, 95\% CI 1.15-1.30) groups compared to the White group (Figure 3, Table 4). When broken down into the 16 categories, the IRR for influenza/ILI was higher in all groups included in the South Asian, Black and Mixed broad ethnic classifications, with the highest IRR in the Bangladeshi group (2.26, 95\% CI 2.05-2.49). After additional adjustment for deprivation and region, results remained similar.

For ARI, the IRR was higher in the South Asian group (1.07, 95\% CI 1.05-1.09) when compared to the White group, but lower in the Black $(0.81,95 \%$ CI $0.80-0.83)$, Mixed
(0.84, 95\% CI $0.81-0.88)$ and Other $(0.65,95 \%$ CI $0.63-0.67)$ groups. Using the 16 categories, the IRR for ARI was only higher for the Pakistani $(1.42,95 \%$ CI 1.37-1.46) and Bangladeshi (1.41, 95\% CI 1.33-1.50) groups when compared with the White British group.

\section{Discussion}

We showed an increased rate of influenza/ILI among Black, South Asian and Mixed groups. Specifically, those of Indian, Pakistani, Bangladeshi and African ethnicity had the highest rate compared to the White British group. When using our broader outcome of ARI, we only found an increased rate in the South Asian group with decreased rates in Black, Mixed and Other groups.

Our results suggest influenza infection risk differs between White and non-White groups. Such findings are consistent with studies of other acute viral respiratory infections including those which investigated the ethnic disparities in severe influenza outcomes, particularly during the $2009 \mathrm{H} 1 \mathrm{~N} 1$ pandemic $^{5,6}$ as well as studies of COVID-19 infection risk and severe outcomes ${ }^{1}$.

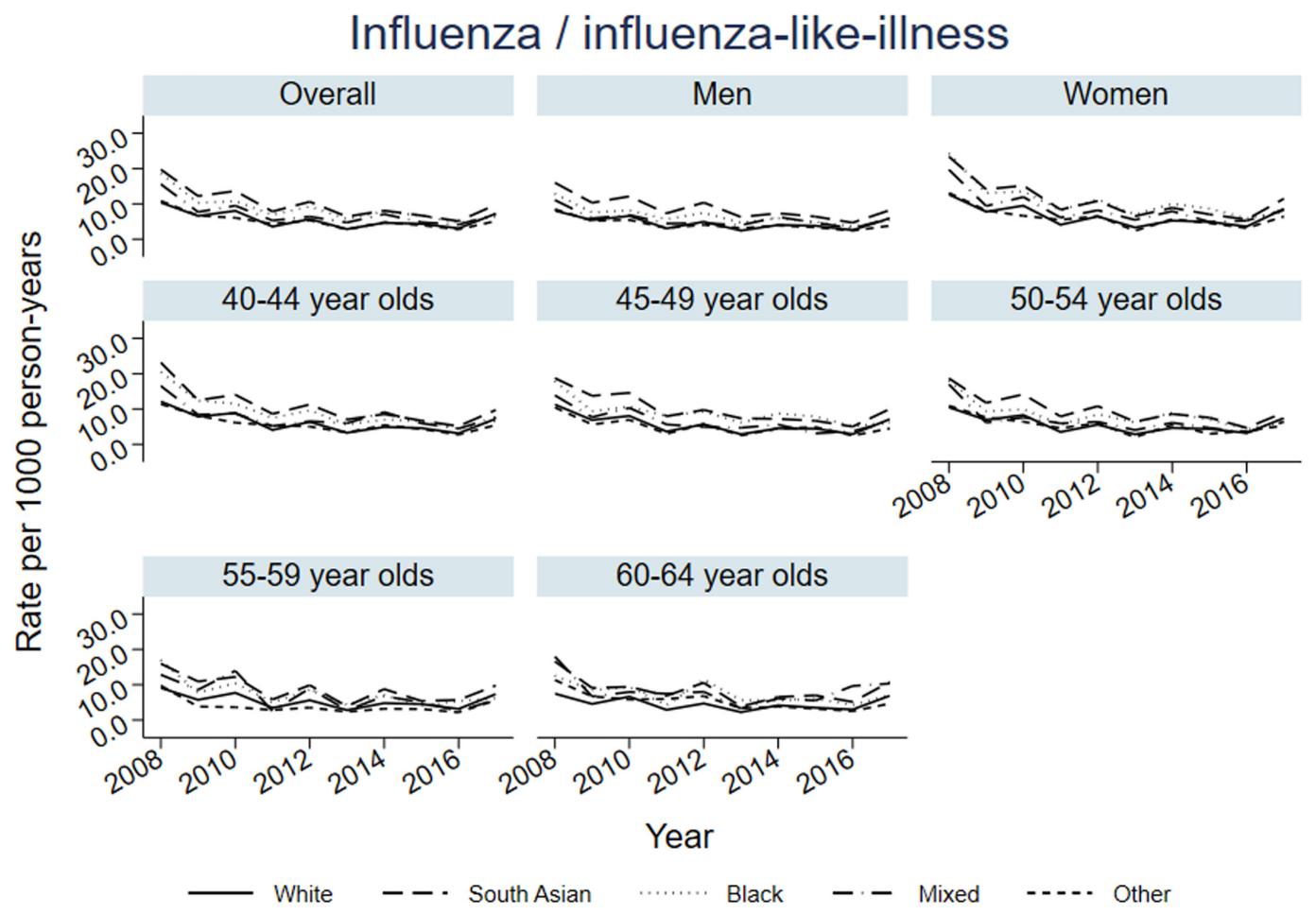

Figure 2. Overall, age- and sex-stratified annual incidence rates for influenza / influenza-like illness by ethnic group. 


\begin{tabular}{|c|c|c|c|c|c|c|c|c|c|c|c|c|c|c|c|c|c|c|c|c|c|c|c|c|}
\hline \multirow{5}{*}{ 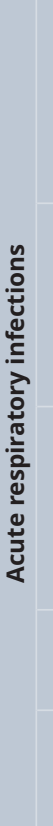 } & 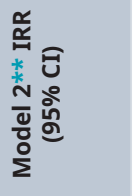 & & 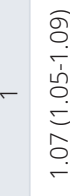 & 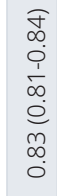 & 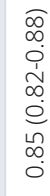 & $\begin{array}{l}\widehat{\sigma} \\
0 \\
0 \\
\dot{1} \\
0 \\
0 \\
\hat{e} \\
\hat{0} \\
0\end{array}$ & & 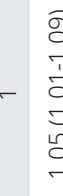 & 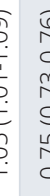 & & 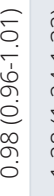 & 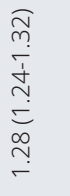 & 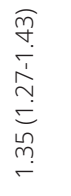 & 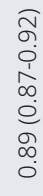 & $\begin{array}{l}\bar{\alpha} \\
\infty \\
0 \\
0 \\
0 \\
0 \\
0 \\
\infty \\
0 \\
0\end{array}$ & 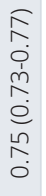 & 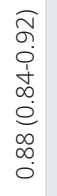 & 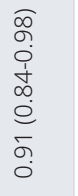 & 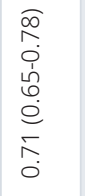 & $\begin{array}{l}0 \\
\circ \\
o \\
0 \\
\frac{1}{\infty} \\
0 \\
\infty \\
\infty \\
\infty \\
0 \\
0\end{array}$ & $\begin{array}{l}\widehat{N} \\
\infty \\
0 \\
\hat{\Lambda} \\
\hat{N} \\
\hat{\infty} \\
\infty \\
\hat{0} \\
0\end{array}$ & 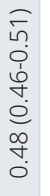 & 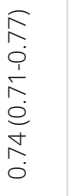 & \\
\hline & 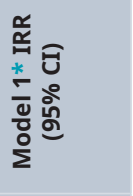 & & 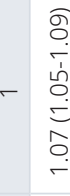 & $\begin{array}{l}\widehat{\alpha} \\
\infty \\
0 \\
0 \\
\infty \\
\infty \\
\stackrel{0}{\infty} \\
\infty \\
0 \\
0\end{array}$ & $\begin{array}{l}0 \\
\infty \\
\infty \\
0 \\
0 \\
\infty \\
\infty \\
0 \\
0 \\
0 \\
0 \\
0\end{array}$ & $\begin{array}{l}\sqrt{6} \\
0 \\
0 \\
0 \\
0 \\
0 \\
0 \\
0 \\
0 \\
0\end{array}$ & & $\begin{array}{r}8 \\
- \\
- \\
0 \\
0 \\
0 \\
0 \\
0 \\
0\end{array}$ & 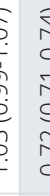 & & 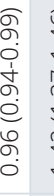 & 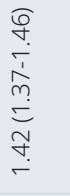 & 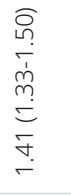 & $\begin{array}{l}\widehat{\sigma} \\
\infty \\
0 \\
\vdots \\
\infty \\
0 \\
0 \\
\infty \\
0 \\
0\end{array}$ & $\begin{array}{l}\widehat{0} \\
0 \\
0 \\
0 \\
\hat{1} \\
0 \\
0 \\
0 \\
0 \\
0\end{array}$ & 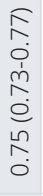 & $\begin{array}{l}\text { f } \\
0 \\
0 \\
0 \\
0 \\
0 \\
0 \\
0 \\
0 \\
0\end{array}$ & 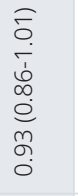 & $\begin{array}{l}\widehat{o} \\
\infty \\
o \\
0 \\
0 \\
0 \\
0 \\
\\
\hat{0} \\
0\end{array}$ & 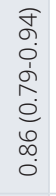 & 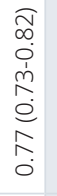 & 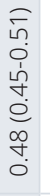 & 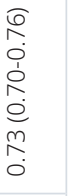 & \\
\hline & 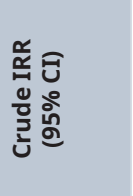 & & 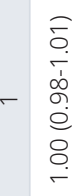 & $\begin{array}{l}0 \\
0 \\
0 \\
0 \\
1 \\
\hat{n} \\
0 \\
0 \\
0 \\
0 \\
0\end{array}$ & 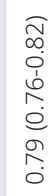 & $\begin{array}{l}f \\
0 \\
0 \\
\vdots \\
0 \\
0 \\
0 \\
0 \\
0 \\
0\end{array}$ & & 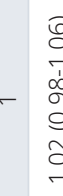 & 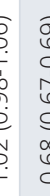 & & 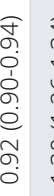 & 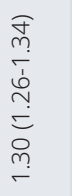 & 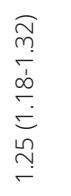 & 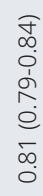 & $\begin{array}{l}\widehat{O} \\
\infty \\
0 \\
\dot{1} \\
\hat{2} \\
\hat{0} \\
\hat{1} \\
0\end{array}$ & $\begin{array}{l}\overline{1} \\
\text { i. } \\
\vdots \\
0 \\
0 \\
0 \\
0 \\
0 \\
0 \\
0\end{array}$ & $\begin{array}{l}0 \\
\infty \\
\infty \\
0 \\
i \\
\infty \\
0 \\
0 \\
0 \\
\infty \\
0 \\
0\end{array}$ & $\begin{array}{l}\text { గn } \\
0 \\
0 \\
\vdots \\
\infty \\
0 \\
\infty \\
\infty \\
\infty \\
0 \\
0\end{array}$ & $\begin{array}{l}\widehat{n} \\
\hat{0} \\
i \\
\dot{1} \\
0 \\
0 \\
0 \\
0 \\
0\end{array}$ & $\begin{array}{l}\widehat{c} \\
\infty \\
0 \\
+ \\
+ \\
0 \\
0 \\
0 \\
0 \\
0 \\
0\end{array}$ & $\begin{array}{l}\mathbb{R} \\
0 \\
0 \\
0 \\
0 \\
0 \\
\stackrel{0}{0} \\
\hat{0} \\
0\end{array}$ & 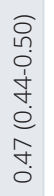 & $\begin{array}{l}\widehat{N} \\
\hat{i} \\
\dot{1} \\
b \\
0 \\
0 \\
0 \\
0 \\
0 \\
0\end{array}$ & \\
\hline & 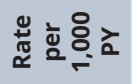 & ल) & & ્ֶڤ & $\stackrel{\sigma}{\stackrel{\sigma}{N}}$ & $\stackrel{n}{\bar{N}}$ & & 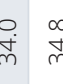 & $\begin{array}{l}0^{\circ} \\
\stackrel{1}{n}\end{array}$ & f & صn. & $\stackrel{\sim}{\sim}$ & $\stackrel{a}{\stackrel{\sim}{\sim}}$ & ฝี & $\underset{\sim}{\tilde{m}}$ & 字 & $\begin{array}{l}\stackrel{\infty}{r} \\
\stackrel{\gamma}{q}\end{array}$ & $\begin{array}{l}\stackrel{\sim}{\infty} \\
\infty\end{array}$ & $\stackrel{\infty}{\stackrel{\infty}{N}}$ & $\stackrel{\llcorner}{\stackrel{\sim}{\sim}}$ & $\hat{\grave{N}}$ & $\begin{array}{l}\stackrel{\varphi}{\dot{\varphi}} \\
\leftarrow\end{array}$ & $\stackrel{m}{\stackrel{\sim}{\sim}}$ & \\
\hline & 营 & 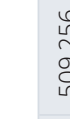 & 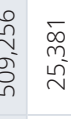 & $\begin{array}{l}\infty \\
\infty \\
0 \\
\leftarrow \\
\leftarrow\end{array}$ & 隽 & 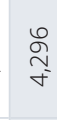 & & 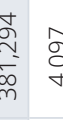 & 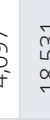 & & مे & ఫु & $\underset{\wp}{\sim}$ & 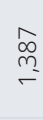 & 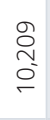 & 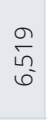 & 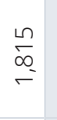 & $\begin{array}{l}\infty \\
\infty \\
\infty \\
0 \\
0\end{array}$ & $\begin{array}{l}\text { no } \\
0 \\
\text { in }\end{array}$ & 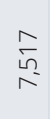 & $\begin{array}{c}\hat{o} \\
m \\
m\end{array}$ & 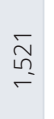 & हे & 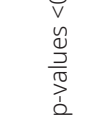 \\
\hline \multirow{8}{*}{ 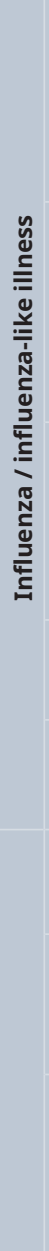 } & 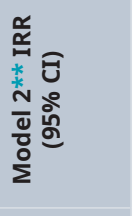 & 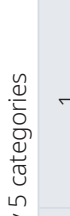 & 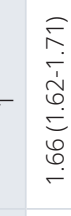 & 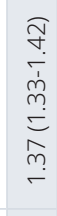 & 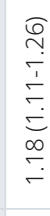 & $\begin{array}{l}\widehat{m} \\
\text { o } \\
0 \\
\infty \\
\infty \\
0 \\
\infty \\
\infty \\
\infty \\
0\end{array}$ & 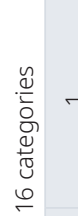 & 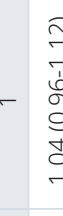 & 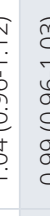 & & 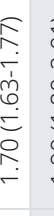 & 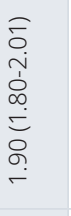 & 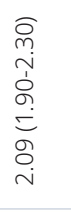 & 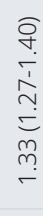 & 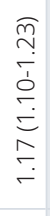 & 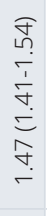 & 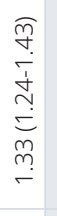 & 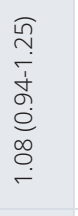 & 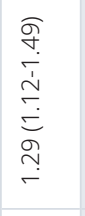 & 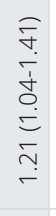 & 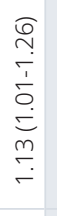 & $\begin{array}{l}0 \\
0 \\
0 \\
0 \\
0 \\
\hat{n} \\
0 \\
0 \\
\hat{n} \\
0\end{array}$ & 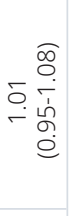 & 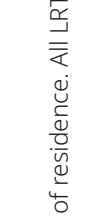 \\
\hline & 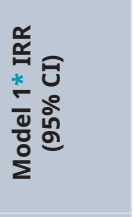 & 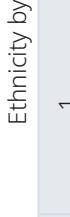 & 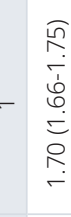 & 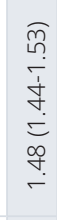 & 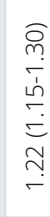 & $\begin{array}{l}\widehat{\Omega} \\
\circ \\
0 \\
0 \\
\omega \\
\infty \\
0 \\
0 \\
\circ \\
0 \\
0\end{array}$ & - & $\begin{array}{r}5 \\
5 \\
- \\
0 \\
0 \\
0 \\
0 \\
0\end{array}$ & 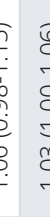 & & 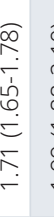 & 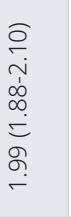 & 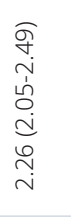 & 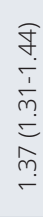 & 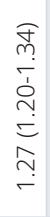 & 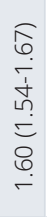 & 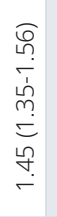 & 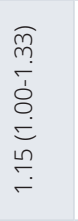 & 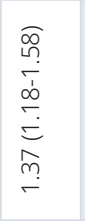 & 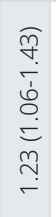 & 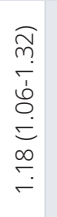 & 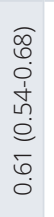 & 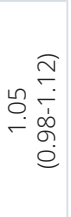 & 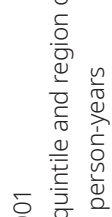 \\
\hline & 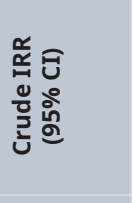 & & 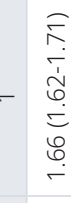 & 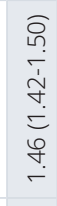 & 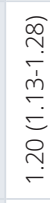 & 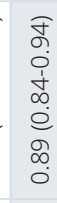 & & 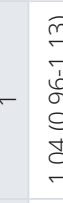 & 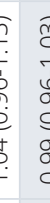 & & 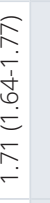 & 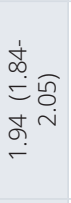 & 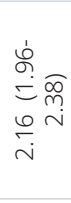 & 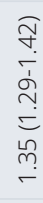 & 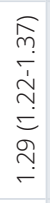 & 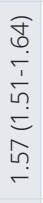 & 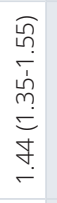 & 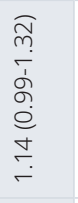 & 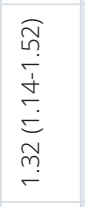 & 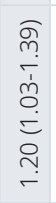 & 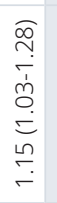 & 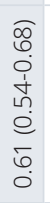 & 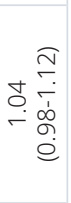 & 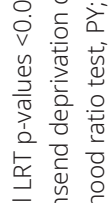 \\
\hline & 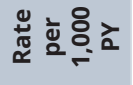 & is & $\stackrel{\sigma}{\sigma}$ & $\stackrel{\nabla}{+}$ & $\stackrel{\sigma}{b}$ & in & & is & Di & & అ. & $\stackrel{\circ}{\wedge}$ & $\stackrel{\sigma}{\varphi}$ & $\stackrel{\bullet}{\circ}$ & $\stackrel{\infty}{\sigma}$ & 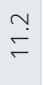 & 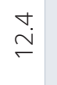 & $\stackrel{\infty}{\sim}$ & $\stackrel{+}{\stackrel{r}{r}}$ & নं & $\stackrel{+}{\infty}$ & $\stackrel{\llcorner}{m}$ & $\stackrel{\circ}{\circ}$ & \\
\hline & 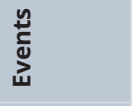 & 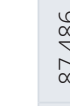 & 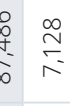 & $\stackrel{\infty}{m}_{\text {ஸे }}$ & $\stackrel{\text { ్ }}{\leftarrow}$ & $\stackrel{ }{\frac{0}{\check{E}}}$ & 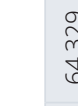 & 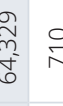 & 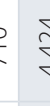 & & $\stackrel{\circ}{\sim}$ & $\stackrel{\circ}{\grave{v}}$ & $\stackrel{\infty}{\infty}$ & ్ָ & 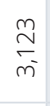 & $\underbrace{}_{\tilde{\sigma}}$ & in & $\stackrel{\substack{+\infty}}{-}$ & 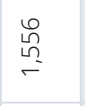 & $\begin{array}{l}\bar{\delta} \\
\infty \\
i \\
i\end{array}$ & ฝू & $\underset{\sim}{\mathbb{N}}$ & స̃ & \\
\hline & $\begin{array}{l}\text { む̊ㅇ } \\
\text { ๖. }\end{array}$ & $\begin{array}{l}0 \\
\text { in } \\
\text { in }\end{array}$ & 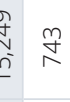 & ్ָరి & ㅆ & $\underline{8}$ & & 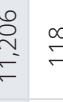 & $=$ & & $\stackrel{\sim}{m}$ & $\stackrel{\infty}{\sim}$ & $\grave{\sim}$ & 出 & $\hat{\bar{m}}$ & 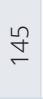 & 广 & $\stackrel{\text { g }}{\sim}$ & 오 & \&̊ & $\stackrel{m}{r}$ & б̆ & $\underline{\sigma}$ & \\
\hline & 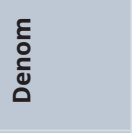 & 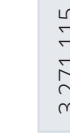 & 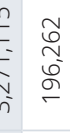 & 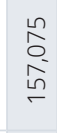 & 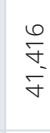 & $\frac{8}{g}$ & 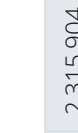 & 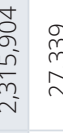 & ?̂. & & $\begin{array}{l}\text { ô } \\
\text { ó } \\
\text { ò }\end{array}$ & 會 & 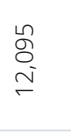 & $\begin{array}{l}\text { बे } \\
\text { ثे } \\
\text { î }\end{array}$ & 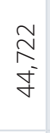 & 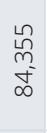 & \begin{tabular}{l}
$\infty$ \\
$\stackrel{\circ}{\circ}$ \\
\multirow{\sim}{\sim}{}
\end{tabular} & 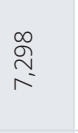 & 苂 & 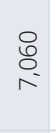 & 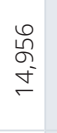 & 昰 & $\begin{array}{c}\bar{a} \\
\text { ó } \\
0\end{array}$ & \\
\hline & & $\frac{d}{3}$ & 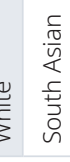 & $\begin{array}{l}\frac{r}{\tilde{U}} \\
\frac{\pi}{\omega}\end{array}$ & ix & 壱 & & 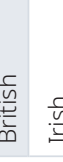 & $\bar{E}$ & & $\underline{0}$ & 离 & $\begin{array}{l}\frac{8}{0} \\
\frac{\pi}{0} \\
\frac{0}{0} \\
\infty\end{array}$ & 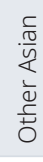 & 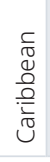 & 胥 & 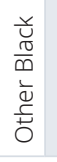 & 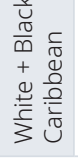 & 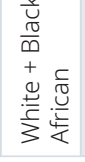 & 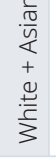 & 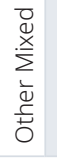 & & 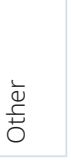 & $E x$ \\
\hline
\end{tabular}




\section{Influenza / influenza-like-illness}

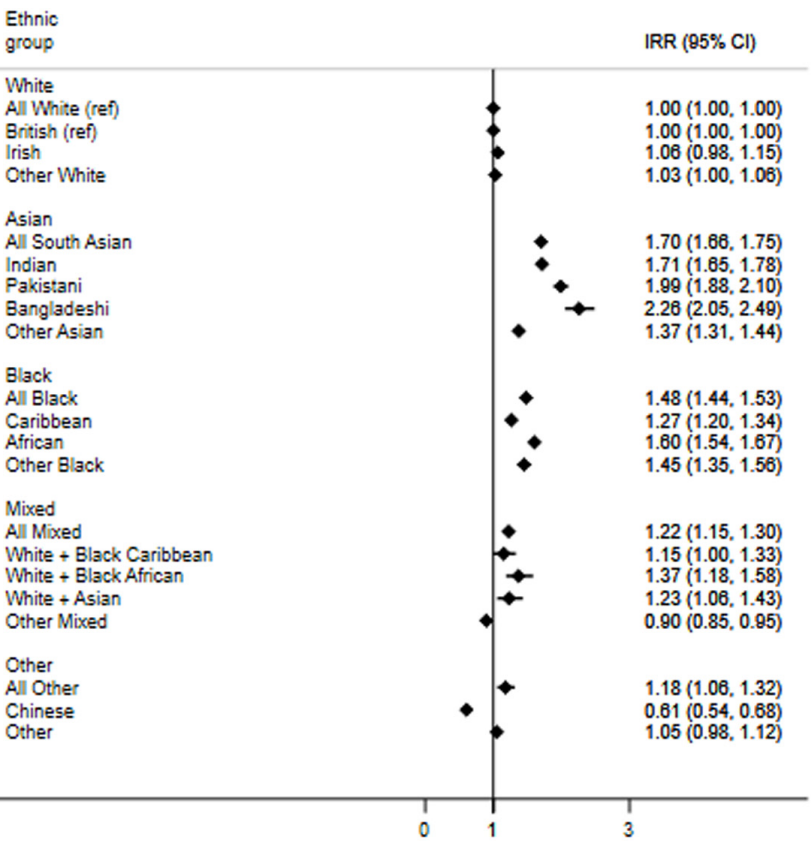

\section{Acute respiratory infection}

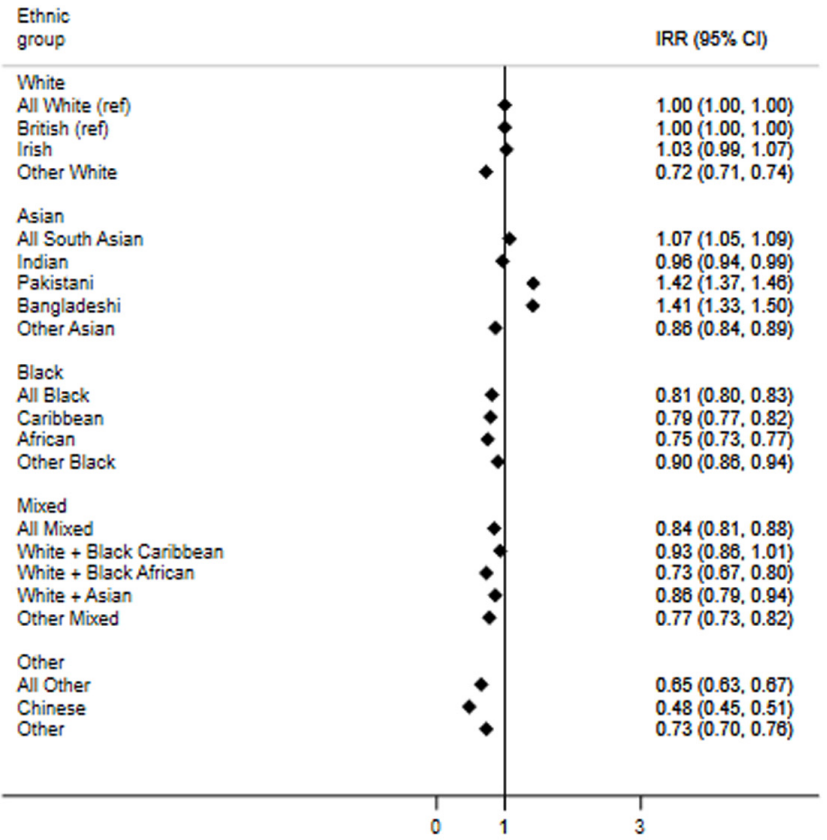

Figure 3. Ethnic differences in the incidence ratio risks of influenza / influenza-like illness and acute respiratory infections. All White is the reference category for comparison of ethnicity in 5 categories. British is the reference category for comparison of ethnicity in 16 categories. Models were adjusted for 5-year age band, sex, year.

Our study was conducted among patients not eligible for vaccination, and so disparities cannot be explained by differences in vaccine uptake or effectiveness: there are potentially even larger ethnic differences in influenza incidence among those eligible for influenza vaccine due to inequalities in chronic disease patterns. Since social mixing and household contact are important considerations for influenza/ILI transmission our findings are relevant to the whole population. People of non-white ethnicity tend to live in larger, multi-generational households with extended kinship and social networks ${ }^{9,10}$. Therefore, understanding ethnic disparities in respiratory infections across both high- and low-risk populations remains important for preventing hospitalizations.

Here we have presented results of a large population-based cohort study using nationally representative data. Excluding patients eligible for influenza vaccination due to chronic medical conditions should have reduced confounding. Nevertheless, our study may be impacted by some limitations. Under-diagnosis of health conditions may differ by ethnicity, with people from some ethnic groups less likely to be excluded from our study population but more likely to have an undiagnosed, and therefore unmanaged condition, which may affect influenza risk. Ethnicity may be less well recorded in GP records for individuals without a chronic condition requiring frequent consultation, but financial incentivization between 2006-2011 boosted completion in GP records. Using hospital data boosted the completeness of ethnicity recording in our study population from $74 \%$ to $88 \%$. Influenza infection in our study was based on clinical diagnosis. Clinically identified influenza depends not only on healthcare attendance but also clinical coding practices, both of which may be associated with ethnicity. However, our results are consistent with other studies which used laboratory-confirmed measures of acute viral respiratory infections ${ }^{1,5,6}$. Our differing results for influenza/ILI and ARI outcomes may be attributable to the lack of specificity of ARI codes for influenza. We excluded individuals with known risk factors for influenza; it may be that other conditions are relevant risk factors for ARI generally.

Ethnic inequalities in the incidence of respiratory infections could arise because of differences in risk of exposure, driven by factors such as occupation and household composition ${ }^{11}$, as well as inequalities in access to care. We excluded children who are a key driver for influenza transmission; examining ethnic inequalities for infection risk in children is an area for future research. Unequal access to treatments will also affect the likelihood of adverse outcomes after infection.

The COVID-19 pandemic has highlighted ethnic inequalities in infection risk, which our study found are also present for influenza. This reinforces the urgency of addressing lower influenza vaccine uptake among minority ethnic groups, which could be combined with public health interventions to promote equal uptake of COVID-19 vaccination ${ }^{12}$. 


\section{Data availability}

Source data

The patient data used in this study are supplied from Clinical Practice Research Datalink (CPRD; www.cprd.com) but restrictions apply to the availability of these data, which were obtained under licence from the UK Medicines and Healthcare products Regulatory Agency, and so are not publicly available. For re-using these data, an application must be made directly to CPRD. Instructions for how to submit an application and the conditions under which access will be granted are explained at https://www.cprd.com/ research-applications.

\section{Ethical approval}

The study was approved by the CPRD Independent Scientific Advisory Committee (Protocol number: 19_209A2) and the
London School of Hygiene and Tropical Medicine Research Ethics Committee (Reference: 17894).

\section{Acknowledgements}

This study is based on anonymized data from the Clinical Practice Research Datalink obtained under licence from the UK Medicines and Healthcare products Regulatory Agency. The data is provided by patients and collected by the national health service (NHS) as part of their care and support. The interpretation and conclusions contained in this study are those of the authors alone.

We would like to thank Vanessa Saliba, Consultant Epidemiologist in the department of Immunisation and Countermeasures at Public Health England, for her review of our study protocol and manuscript.
1. Mathur R, Rentsch CT, Morton CE, et al.: Ethnic differences in COVID-19 infection, hospitalisation, and mortality: an OpenSAFELY analysis of 17 million adults in England. medRxiv. 2020; 2020.09.22.20198754. Publisher Full Text

2. Sze $S$, Pan $D$, Nevill CR, et al.: Ethnicity and clinical outcomes in COVID-19: A systematic review and meta-analysis. EClinicalMedicine. 2020; 29: 100630. PubMed Abstract | Publisher Full Text | Free Full Text

3. Public Health England: Influenza: the green book, chapter 19. 2020. Reference Source

4. Public Health England: Seasonal flu vaccine uptake in GP patients: monthly data, 2020 to 2021 Reference Source

5. Zhao H, Harris RJ, Ellis J, et al.: Ethnicity, deprivation and mortality due to 2009 pandemic influenza $A(H 1 N 1)$ in England during the 2009/2010 pandemic and the first post-pandemic season. Epidemiol Infect. 2015; 143(16): 3375-83. PubMed Abstract | Publisher Full Text

6. Chandrasekhar R, Sloan C, Mitchel E, et al: Social determinants of influenza hospitalization in the United States. Influenza Other Respi Viruses. 2017; 11(6): 479-88.

PubMed Abstract | Publisher Full Text | Free Full Text
7. Herrett E, Gallagher AM, Bhaskaran K, et al.: Data Resource Profile: Clinical Practice Research Datalink (CPRD). Int J Epidemiol. 2015; 44(3): 827-36. PubMed Abstract | Publisher Full Text | Free Full Text

8. Wolf A, Dedman D, Campbell J, et al.: Data resource profile: Clinical Practice Research Datalink (CPRD) Aurum. Int J Epidemiol. 2019; 48(6): 1740-1740g. PubMed Abstract | Publisher Full Text | Free Full Text

9. Mathur R, Bear L, Khunti K, et al.: Urgent actions and policies needed to address COVID-19 among UK ethnic minorities. Lancet. 2020; 396(10266): 1866-1868.

PubMed Abstract | Publisher Full Text | Free Full Text

10. Scientific Advisory Group for Emergencies: Housing, household transmission and ethnicity, 26 November 2020. GOV.UK. 2020. Reference Source

11. Nafilyan V, Islam N, Ayoubkhani D, et al.: Ethnicity, Household Composition and COVID-19 Mortality: A National Linked Data Study. medRxiv. 2020; 2020.11.27.20238147 Publisher Full Text

12. Ekezie W, Czyznikowska BM, Rohit S, et al:: The views of ethnic minority and vulnerable communities towards participation in COVID-19 vaccine trials. J Public Health (Oxf). 2020; fdaa196.

PubMed Abstract | Publisher Full Text | Free Full Text 


\section{Open Peer Review}

\section{Current Peer Review Status:}

\section{Version 1}

Reviewer Report 08 March 2021

https://doi.org/10.21956/wellcomeopenres.18323.r42945

(C) 2021 Cowling B. This is an open access peer review report distributed under the terms of the Creative Commons Attribution License, which permits unrestricted use, distribution, and reproduction in any medium, provided the original work is properly cited.

\section{Benjamin J. Cowling}

WHO Collaborating Centre for Infectious Disease Epidemiology and Control, School of Public Health, The University of Hong Kong, Hong Kong, China

This is a nice study using electronic health records, with clear methodology and findings.

Influenza/ILI in CPRD is mainly ILI, so I am not sure your conclusion "Our results suggest influenza infection risk..." might be fairer to discuss ILI than influenza virus infection?

To what extent could access to care also affect comparisons? You are not studying incidence of infections directly, but medically-attended illnesses? On a related note, if sick-notes are required in service sector but less frequently for white collar professions, would it lead to greater consultations among lower income professions?

I saw 12 references in the bibliography and wondered if there might be more (additional) similar studies that it would be relevant to cite here?

Is the work clearly and accurately presented and does it cite the current literature? Yes

Is the study design appropriate and is the work technically sound? Yes

Are sufficient details of methods and analysis provided to allow replication by others? Yes

If applicable, is the statistical analysis and its interpretation appropriate? Yes

Are all the source data underlying the results available to ensure full reproducibility? 


\section{Partly}

Are the conclusions drawn adequately supported by the results?

Yes

Competing Interests: No competing interests were disclosed.

Reviewer Expertise: Infectious disease epidemiology

I confirm that I have read this submission and believe that I have an appropriate level of expertise to confirm that it is of an acceptable scientific standard. 